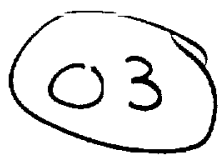

\title{
A Post-Pathfinder Evaluation of Areocentric Solar Coordinates with Improved Timing Recipes for Mars Seasonal/Diurnal Climate Studies
}

\author{
Michael Allison ${ }^{1}$ and Megan McEwen ${ }^{2}$

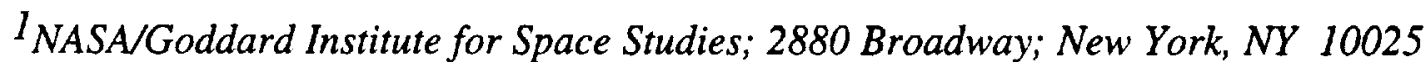 \\ ${ }^{2}$ Department of Astronomy, Columbia University; 550 West 120th Street, New York, NY 10027
}

Prepared for

Planetary and Space Science 


\section{Abstract}

The accurate determination of the Mars pole vector derived from Pathfinder and Viking Lander radio data (Folkner et al., 1997), together with the VSOP87 representation of planetary orbits (Bretagnon and Francou, 1988), have been applied to a new evaluation of the right ascension of the "fictitious mean sun" (FMS) at Mars. With $\Delta \mathrm{t}_{\mathrm{J} 2000}$ the elapsed time in days from the $\mathrm{J} 2000$ epoch (J.D.2451545.0 $0^{\mathrm{TT}}$ ), $\quad \alpha_{\mathrm{FMS}}=270^{\circ} .3863+0.52403840(\%) \cdot \Delta \mathrm{t}_{\mathrm{J} 2000}$ $-4 \times 10^{-13}\left({ }^{\circ} / \mathrm{d}^{2}\right) \cdot \Delta \mathrm{t}^{2}{ }_{\mathrm{J} 2000}$ represents a best least-squares quadratic fit of the FMS, including aberration, to each instance of the four equinox and solstice passages for each of 134 Mars orbits spanning the calendar years 1874-2127. The implied tropical orbit period for Mars, $686.9726^{\mathrm{d}}$, closely agrees with the recent evaluations by Suran (1997) and Allison (1997). Together with the Pathfinder radio determination of the Mars sidereal rotation, the derived FMS rate corresponds to a mean solar day (or "sol") of $1.027491251^{\mathrm{d}}$. The new FMS determination would serve to define the Mean Solar Time at Mars to the nearest tenth-second, according to historical conventions originally established for terrestrial time keeping, once the Mars prime meridian defined by the crater Airy- 0 is navigated to the same accuracy. For convenient reference to current epochs, 2000 Jan 06 00:00 UTC (= MJD 51549.000 UTC) corresponds to a coincidence of $\alpha_{F M S}$ and the rotation angle of the crater Airy-0 measured with respect to the Mars equinox (i.e. "mean solar midnight" on the planet's prime meridian), to within the current uncertainty of several seconds in the locational definition of the planet's cartographic grid. As a further result of the analysis, the consistently derived Mars obliquity of date is $\varepsilon=25^{\circ} .192$ $+3.45 \times 10^{-7}(\% / \mathrm{d}) \cdot \Delta \mathrm{t}_{\mathrm{J} 2000}$. An improved analytic recipe for the calculation of the solar areocentric longitude $\left(\mathrm{L}_{\mathrm{s}}\right)$ of Mars to an accuracy of $0^{\circ} .01$ is also provided, accounting for the primary perturbations of Earth, Jupiter, and Venus, which may in turn be applied to an efficient evaluation of Mars local true solar time (LTST) to within the uncertainty of the inertial position of the Mars prime meridian. For specific applications to the data archives for landed Mars spacecraft, simple conversion formulae are given for the determination of the Viking "Local Lander Time" and the Pathfinder "Local True Solar Time" in terms of the terrestrial calendar date and UTC. 


\section{Introduction}

Mars, more than any other planet in the Solar System, exhibits a pronounced and pervasive response to both its diurnal and seasonal modulation of temperature, wind, and surface pressure, with attendant variations in water vapor, atmospheric opacity, boundary layer, ground frost, and polar caps. The radiative cooling time of the Mars atmosphere is more than an order of magnitude shorter than that for the Earth and the eccentricity of the Mars orbit is over five times larger. The fast radiative cooling time and the approximate alignment of the Mars northern winter solstice with the planet's perihelion imply an exaggerated response to solar forcing. The hourly and seasonal timing of the apparent solar position on the planet is therefore a critical issue for Mars geophysical/climate studies, as attested by various reports of lander spacecraft data in reference to both the areocentric solar longitude and the "local lander time" (e.g. Colburn et al., 1989) or the "true local solar time" (e.g. Golombek et $a l ., 1997)$. Yet the only generally accessible sources of precise if still provisional definitions of Martian time coordinates appear to be a NASA technical memorandum (Kaplan, 1988) and a single short paper in the refereed literature (Allison, 1997). The consideration of more accurate definitions of Mars solar time keeping commends a recollection of the original establishment of terrestrial chronological standards.

From 1925 until 1959, Greenwich Mean Time was defined as 12 hours + the Greenwich hour angle of a point on the Earth's equator whose right ascension, measured from the mean equinox of date, was

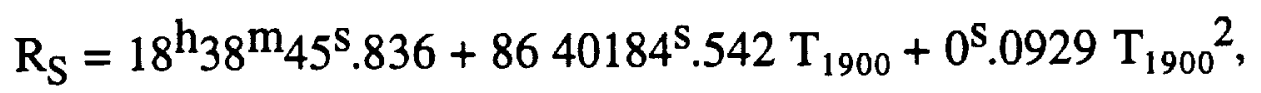

with $T_{1900}$ the number of Julian centuries of 36525 days elapsed since the epoch of Greenwich mean noon on 1900 January 0.5 . The assumed expression for $R_{s}$ was that given by Simon Newcomb (1895) for the right ascension (including aberration) of the "Fictitious Mean Sun" (FMS), an idealized fiducial reference intended to match as closely as possible the uniform motion of the apparent mean solar longitude. Following the realization of the Earth's variable rotation with its lunar-tidal despinning, a distinction was made between Ephemeris Time (ET), 
for which Newcomb's $T$ was interpreted as the independent variable $T_{E}$ of the Earth's (tropical) orbital revolution, and Universal Time (UT), with a variably advancing $T_{U}$ now evaluated in practice by a variety of methods including optical transit observations of stars, lunar laser ranging, and Very Long Baseline Interferometry of cosmic radio sources.

Beginning in 1955, atomic clocks became available in several countries and since 1971 the accepted international time standard has been Temps Atomique International (TAI), with Terrestrial Time (TT), the successor to ET, now given as $\mathrm{TT}=\mathrm{ET}=\mathrm{TAI}+32.18^{\mathrm{S}}$. (The difference $\mathrm{ET}-\mathrm{TAI}=32.18^{\mathrm{S}}$ corresponds to the evaluated departure $\Delta \mathrm{T}$ of Ephemeris Time from Universal Time on 1958 January 0.) Although the Fictitious Mean Sun has consequently been abandoned for precision time-keeping, Newcomb's mean solar coordinate formulas served not only the original calibration of the 24 hour day, but also the original definition of both the J1900 ephemeris epoch and the ephemeris second. Although the System International (SI) second is now defined as 9,192,631,770 periods of the hyperfine transition of the ground state of cesium-133, this standard was originally calibrated by Markowitz et al. (1958) to match the astronomically determined value of the ephemeris second, itself established in reference to Newcomb's mean solar coordinate formulas as "la fraction 1/31556925.9747 de l'année tropique pour 1900 janvier 0 à 12 heures de temps des ephemerides" (Comité International de Poids et Mesures, 1957). A further account of the development of modern timekeeping standards and their ongoing reform in accommodation of general relativistic effects is given by Seidelmann and Fukushima (1992).

Although there are as yet no internationally accepted Martian time standards, it may be supposed that these will eventually be referenced to some appropriately accurate determination of the planet's mean solar coordinates. Provisional determinations of the FMS at Mars, as recorded in internal office memoranda at the Jet Propulsion Laboratory (e.g. Blume, 1986; Lee, 1995), have been applied to the dafinition of local solar time for Mars spacecraft operations and data archival. These have been based on fits to computational ephemerides over 
short-term intervals comparable to their intended use for specific flight missions, however, and are not available in the open literature. A 33yr cycle of $\sim 0^{\circ} .002$ perturbations in the Mars orbital longitude by Venus, for example, suggests that a precise and temporarily extensive calibration of the FMS should be fitted to at least several tens of orbits. Allison (1997) presented an independent estimate of the FMS at Mars as fitted to 120 orbits, but to a precision of only $0^{\circ} .01$, corresponding to a definition of the mean solar time to within $2.4 \mathrm{sec}$.

It should be acknowledged that any adopted synchronization of "mean solar time" is largely a matter of convenience. As noted by Woolard and Clemence (1966), "a measure of mean solar time which would depart too far from the average of the apparent solar measure would be inconvenient, and if too discordant would fail to serve practical purposes satisfactorily, but still would not be logically erroneous..." Given the reported $\sim 0^{\circ} .03$ discrepancy between the USGS cartographic and "inertial" Mars longitudes (cf. Golombek, 1997), the quest for much further precision in the evaluated FMS may seem "academic." Perhaps in the not too distant future, Martian Coordinate Time will be established by synchronization to a landed atomic clock or an ultra-stable rubidium oscillator. But while the calibration of Mars/solar coordinates to the implied milliarc second accuracy of Newcomb's FMS formula may never be necessary, it seems appropriate to apply the available data for the Mars orbit and rotation to an assessment of the planet's FMS to the attempted $\left(0^{\circ} .001\right)$ precision of the IAU definition of the Mars prime meridian (Davies et al., 1996). The new determination by Folkner et al. (1997) of the Mars pole vector from Pathfinder and Viking lander radio tracking data now makes possible a new calibration of the Fictitious Mean Sun at Mars over century intervals to a precision of better than $0^{\circ} .001$, as appropriate to a split (0.2) second definition of a mean solar time analogous to terrestrial conventions. The analysis provides an accurate evaluation of the tropical year and mean solar day at Mars, as well as a new calibration of the Mars obliquity of date. The new FMS calibration also serves the ready evaluation of a simple series representation of the areocentric solar longitude and related solar illumination quantities to an accuracy better than $0^{\circ} .01$. 


\section{Planetocentric Solar Coordinates}

Figure 1 illustrates the definition of various planetocentic solar coordinates in application to seasonal/hourly timing. The standard index for planetary seasons is the planetocentric orbital longitude of the Sun $\mathrm{L}_{\mathrm{S}}$, measured eastward along the planet's orbital plane from its vernal equinox (the instantaneous ascending node defined by the intersection of the planet's orbit and equator). For the Earth, $\mathrm{L}_{\mathrm{S}}$ is just the ecliptic longitude of the Sun. For Mars, $\mathrm{L}_{\mathrm{S}}$ is commonly referred to as the areocentric longitude of the Sun. Hourly solar timing must account for the true solar right ascension $\alpha_{S}$ while seasonal climate variations are largely controlled by the planetocentric solar declination $\delta_{S}$. As the indicated legs of the right spherical triangle with hypotenuse $L_{S}$, these are given as $\alpha_{S} \equiv \tan ^{-1}\left(\cos \varepsilon \cdot \tan L_{S}\right)$ and $\delta_{S} \equiv \sin ^{-1}\left(\sin \varepsilon \cdot \sin L_{S}\right)$, where $\varepsilon$ is the obliquity (the inclination of the planet's orbit to its equator).

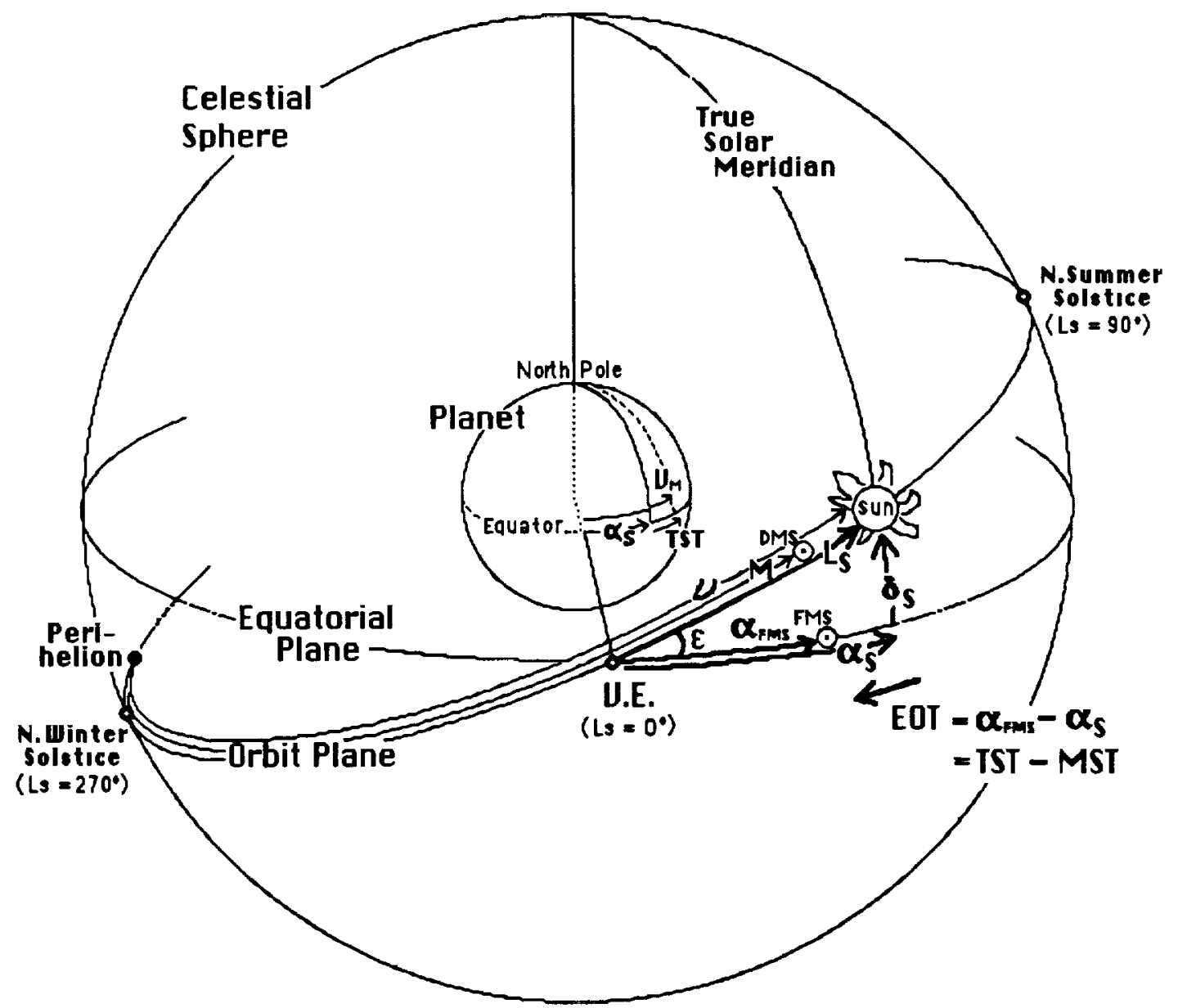

Figure 1. Planetocentric solar coordinates, the Fictitious Mean Sun (FMS), the Equation of Time (EOT) and their relation to the true and mean orbit anomalies. 
The difference between the right ascension with respect to the planet's vernal equinox of a given planetary longitude, and the evaluated right ascension of the true Sun constitutes the measure of "solar time." According to conventional usage, the solar hour angle or true solar time (TST) on the planet, as referred to some longitude or meridian with a right ascension $V_{m}$, is just $\left(V_{m}-\alpha_{S}\right) \times\left(24^{h} / 360^{\circ}\right)+12^{h}$, with the 12 hour offset added for the placement of local (12:00:00) "noon" at the instant when the Sun transits the overhead meridian. While $\mathrm{V}_{\mathrm{m}}$ depends only on the (nearly uniform) planetary rotation and some fiducial cartographic reference such as the "prime meridian," $\alpha_{S}$ advances unevenly, both as a result of the planet's eccentric orbit and the obliquity of its rotational plane.

Mean solar time (MST) is established by a mathematical formula prescribing the uniform advance of an imaginary point on the celestial equator known as the Fictitious Mean Sun (FMS). As established for terrestrial time-keeping, the FMS is defined so that its angular displacment along the equator from the vernal equinox (V.E.) is matched as closely as possible to the angular displacement with respect to the V.E. (including aberration) of a dynamical mean sun (DMS) moving uniformly along the orbital plane. (cf. Woolard and Clemence, 1966; Roy, 1982; Green, 1985.)

The position of the DMS along its orbit as measured with respect to the perihelion is just the classical mean anomaly $M \equiv(L-\bar{\omega})$, where $L$ is the mean longitude and $\Phi$ the longitude of perihelion. As evaluated at a time $t$ after some reference epoch $t_{0}, M$ may be defined as

$$
M \equiv\left[\left(t_{0}-t_{p}\right)+\left(t-t_{0}\right)\right] n_{\text {anom }},
$$

where $t_{p} \equiv t_{0}-M\left(t_{0}\right) / n_{\text {anom }}$ is the indicated time of perihelion passage for the mean-fitted orbit, with $n_{\text {anom }}$ the mean rate of the motion corresponding to the anomalistic period $\tau_{\text {anom }}=360 \% \mathrm{n}_{\text {anom }}$.

The difference $(\mathrm{v}-\mathrm{M})$ between the true anamoly $\mathrm{v}$ measuring the true solar position along the orbital plane and the mean anomaly is the equation of center. Then as shown in Fig.1, the position of the dynamical mean sun with respect to the V.E. as measured along its orbit is just the difference between the areocentric 
solar longitude and the equation of center. And the FMS is by construction displaced from the V.E. along the equator by essentially the same amount. [As discussed by Woolard and Clemence (1966), there is actually a very small divergence between $\alpha_{\text {FMS }}$ and the mean solar longitude (of the DMS) owing to a difference between the secular variations of the general precession in right ascension and longitude as well as an acceleration of the apparent planetocentric motion of the Sun. The resulting discrepancy for Earth-solar coordinates is only about 2 seconds in 1000 years, however, and the same effects for areocentric solar coordinates will be entirely neglected here.] A practical definition of the Fictitious Mean Sun ( $\alpha_{\text {FMS }}$ ) may therefore be given as

$$
\alpha_{\mathrm{FMS}} \equiv \mathrm{L}_{\mathrm{S}}-(v-\mathrm{M})
$$

Since $v \equiv M$ at the instant $t_{p}$ coincident with the perihelion passage, the right ascension of the Fictitious Mean Sun at that point is just the areocentric solar longitude at perihelion. At other times $t$, or after some interval $\left(t-t_{0}\right)$ from the epoch $t_{0}$,

$$
\alpha_{F M S}=L_{S}\left(t_{p}\right)+\left[\left(t_{0}-t_{p}\right)+\left(t-t_{0}\right)\right] n_{\text {trop }}
$$

where $n_{\text {trop }}$ is the rate of the apparent "tropical" mean motion of the Sun corresponding to a tropical period $\tau_{\text {trop }}=360 \% \mathrm{n}_{\text {trop. }}$. To the extent that it appropriately serves the accuracy of a particular fit to the Fictitious Mean Sun as calculated from the orbital ephemerides and a specification of the planet's polar precession, the slow variation of the tropical rate itself can be represented as in Newcomb's terrestrial formula by an expanded polynomial time series, with $\alpha_{\text {FMS }}=\alpha_{\text {FMS }}\left(t_{0}\right)+n_{\text {trop }}\left(t-t_{0}\right)+\left(d n_{\text {trop }} / d t\right) \cdot\left(t-t_{0}\right)^{2}+\ldots .$, where $\alpha_{F M S}\left(t_{0}\right) \equiv$ $\mathrm{L}_{S}\left(\mathrm{t}_{\mathrm{p}}\right)+\left(\mathrm{t}_{0}-\mathrm{t}_{\mathrm{p}}\right) \mathrm{n}_{\text {trop }}$.

As the form of (3) suggests, a rudimentary calibration of the FMS can be established by a fit to the evaluated areocentric solar longitude at successive perihelia over some number of planetary orbits. This was essentially the method adopted by Blume (1986) on behalf of the Mars Observer project, taking a linear fit to $L_{S}\left(t_{p}\right)$ as calculated by the JPL ephemerides for the epochs of the Mars perihelion in the years 1990,1992 , and 1994. A similar method was adopted by 
Lee (1995) for the Mars Surveyor Project, fitting the FMS rate (or tropical mean motion) to successive passages of the Mars vernal equinox for the years 1996, 1998,2000 , and 2002, then calibrating the FMS angle at a particular epoch by its consistent evaluation for an average of the areocentric longitude at the successive Mars perihelia in 1996, 1998, and 2000. These approaches to the FMS determination have the advantage of avoiding any explicit reliance on the classical mean orbital elements, and reflect a small but significant difference between $n_{\text {trop }}$ and $n_{\text {anom }}$ as apparent even over a small number of orbits. Aside from the limits of these fits to their assumed short temporal intervals and their apparent neglect of aberration, however, these approaches do not in principle take the most accurate account of a very small variation for the seasonal repetition of different values of the $\mathrm{L}_{\mathrm{S}}$, as evaluated below.

Alternatively, $\alpha_{\mathrm{FMS}}$ can be fitted to its definition by (2) from an ephemeris calculation of the time of $\mathrm{L}_{\mathrm{S}}$ for different seasons and over several orbits, together with the corresponding evaluation of the equation of center. By a classical result of celestial mechanics for the two-body Kepler motion, $(v-M)$ may be efficiently evaluated by a Fourier-Bessel series for the mean anomaly and the orbital eccentricity e. By a truncation to sixth order in e, for example, of the results given by Taff (1985):

$$
\begin{gathered}
(v-M)=\left(2 e-\frac{1}{4} e^{3}+\frac{5}{96} e^{5}\right) \sin M+\left(\frac{5}{4} e^{2}-\frac{11}{24} e^{4}+\frac{17}{192} e^{6}\right) \sin 2 M+ \\
\left(\frac{13}{12} e^{3}-\frac{43}{63} e^{5}\right) \sin 3 M+\left(\frac{103}{96} e^{4}-\frac{43}{480} e^{6}\right) \sin 4 M+ \\
\left(\frac{1097}{960} e^{5}\right) \sin 5 M+\left(\frac{1223}{960} e^{6}\right) \sin 6 M+O\left(e^{7}\right) .
\end{gathered}
$$

Given an accurate determination of both $\alpha_{\mathrm{FMS}}$ and $\mathrm{M}$ as a function of time, (2) and (4) also provide a moderately accurate recipe for Ls, excluding the N-body perturbations by other solar system objects on the planet's Kepler motion about the Sun, as considered in succeeding sections of this paper.

The difference $\left(\alpha_{F M S}-\alpha_{S}\right)=-\left[(v-M)+\left(\alpha_{S}-L_{S}\right)\right]$ is the Equation of Time (EOT), also representing the difference between the true solar time (TST) and the mean solar time (MST). The difference between the true solar right 
ascension and the planetocentric solar longitude $\left(\alpha_{S}-L_{S}\right)$, referred to by Smart (1962) as the reduction to the equator, can be evaluated by the rapidly converging series

$$
\begin{gathered}
\alpha_{S}-L_{S}=\sum_{n=1}^{\infty} \frac{1}{n}\left(-\tan ^{2} \frac{\varepsilon}{2}\right)^{2 n} \cdot \sin (2 n L s) \\
\approx\left(-\tan ^{2} \frac{\varepsilon}{2} \sin 2 L s+\frac{1}{2} \tan ^{4} \frac{\varepsilon}{2} \sin 4 L s-\frac{1}{3} \tan ^{6} \frac{\varepsilon}{2} \sin 6 L s\right) .
\end{gathered}
$$

Given $L_{S}$, this affords an accurate and efficient evaluation of $\alpha_{S}$, and therefore with (4) the EOT, without the need to test for the proper quadrant of the inverse tangent in the spherical trigonometric definition of the true solar right ascension (and the tangent infinites at Ls $=90$ and $270^{\circ}$ ). For a non-vanishing obliquity, $\alpha_{S}<L_{S}$ between each equinox and the succeeding solstice, while $\alpha_{S}>L_{S}$ between each solstice and the succeeding equinox.

For some purposes it is also useful to develop a representation for the true anomaly in terms of the seasonal index. Upon the elimination of $\alpha_{F M S}$ and $M$ between (1), (2) and (3),

$$
v=\mathrm{L}_{S}-\left[\mathrm{L}_{S P}\left(\mathrm{t}_{0}\right)+\mathrm{n}_{\mathrm{SP}}\left(\mathrm{t}-\mathrm{t}_{0}\right)\right]
$$

where

$$
\begin{aligned}
L_{S P}\left(t_{0}\right) & \equiv L_{S}\left(t_{p}\right)+\left(t_{0}-t_{p}\right) n_{S P} \\
& =\alpha_{F M S}\left(t_{0}\right)-M\left(t_{0}\right)
\end{aligned}
$$

is the areocentric solar longitude at perihelion as adjusted to the epoch $t_{0}$ in proportion to

$$
\mathrm{n}_{\mathrm{SP}} \equiv\left(\mathrm{n}_{\mathrm{trop}}-\mathrm{n}_{\mathrm{anom}}\right)
$$

representing the slow rate of advance of the perihelion with respect to the equinox, as a result of the planet's polar precession. Owing to the small value of $\mathrm{n}_{\mathrm{SP}}$ (roughly $2 \times 10^{-5} \% \mathrm{~d}$ for Mars), $\mathrm{v} \approx \mathrm{L}_{\mathrm{S}}-\mathrm{L}_{\mathrm{SP}}\left(\mathrm{t}_{0}\right)$ for short-term intervals about $\mathrm{t}_{0}$, as often assumed for the evaluation of the time of season by the solution of Kepler's equation (e.g. Atkinson and Gwynne, 1992; Badescu, 1998).

An accurate, closed-form representation of the time of season $L_{s}$ can, however, be derived from the development of the orbital element equations presented here. First solving (2) and (3) for the elapsed time post-perihelion by 
the elimination between them of $\alpha_{F M S}, t=t_{p}+\left[L_{s}-L_{S}\left(t_{p}\right)-(v-M)\right] n_{\text {trop }}$. Then the equation of center as it appears in this expression can be evaluated by its second-order expansion in terms of the true anomaly as $(v-M) \approx$ $2 \mathrm{e} \sin v-\left(3 \mathrm{e}^{2} / 4\right) \sin 2 v$ (cf. Taff, 1985). Then by $(6), \sin v=$ $\sin \left[\mathrm{L}_{\mathrm{s}}-\mathrm{L}_{\mathrm{SP}}-\mathrm{n}_{\mathrm{sp}}\left(\mathrm{t}-\mathrm{t}_{0}\right)\right] \approx \sin \left[\mathrm{L}_{\mathrm{s}}-\mathrm{L}_{\mathrm{SP}}\right]+\left[\mathrm{n}_{\mathrm{sp}}\left(\mathrm{t}-\mathrm{t}_{0}\right)\right] \cdot \cos \left[\mathrm{L}_{\mathrm{s}}-\mathrm{L}_{\mathrm{SP}}\right]$, and similarly $\sin 2 v \approx \sin \left[2\left(\mathrm{~L}_{\mathrm{s}}-\mathrm{L}_{\mathrm{SP}}\right)\right]+\left[2 \mathrm{n}_{\mathrm{sp}}\left(\mathrm{t}-\mathrm{t}_{0}\right)\right] \cdot \cos \left[2\left(\mathrm{~L}_{\mathrm{s}}-\mathrm{L}_{\mathrm{SP}}\right)\right]$. And with $\mathrm{L}_{\mathrm{s}} \rightarrow \mathrm{L}_{\mathrm{s}}+\mathrm{k} \cdot 360^{\circ}$ and $\left(\mathrm{t}-\mathrm{t}_{0}\right) \rightarrow \mathrm{k} \cdot \tau_{\text {trop }}$ for the kth orbit post $\mathrm{t}_{0}$,

$$
\begin{aligned}
& \mathrm{t} \approx \mathrm{t}_{\mathrm{p}}+\left\{\mathrm{L}_{\mathrm{s}}-\mathrm{L}_{\mathrm{S}}\left(\mathrm{t}_{\mathrm{p}}\right)-2 \mathrm{e} \sin \left(\mathrm{L}_{\mathrm{s}}-\mathrm{L}_{\mathrm{SP}}\right)+\frac{3}{4} \mathrm{e}^{2} \sin 2\left(\mathrm{~L}_{\mathrm{s}}-\mathrm{L}_{\mathrm{SP}}\right)\right\} \frac{\tau_{\text {trop }}}{360^{\circ}} \\
& +\mathrm{k} \cdot \tau_{\text {trop }}\left\{1+\left[2 \mathrm{e} \cos \left(\mathrm{L}_{\mathrm{s}}-\mathrm{L}_{\mathrm{SP}}\right)-\frac{3}{2} \mathrm{e}^{2} \cos 2\left(\mathrm{~L}_{\mathrm{s}}-\mathrm{L}_{\mathrm{SP}}\right)\right] \mathrm{n}_{\mathrm{sp}} \frac{\tau_{\text {trop }}}{360^{\circ}}\right\}
\end{aligned}
$$

Although the "tropical year" is often loosely referred to as the average interval from equinox-to-equinox, $\tau_{\text {trop }}$ is more precisely understood to be defined by the formula for the geometric mean longitude of the Sun ( $c f$. p.95 of the Explanatory Suplement to the Astronomical Ephemeris, 1961 or p.157 of Soma and Aoki, 1990). The small variation of the exact mean repetition interval for the seasons is recognized, for example, by the four separate formulas given on p.166 of Meeus (1991) for the occurrence of the equinoxes and solstices on the Earth. The last term in (9), multiplied by $k$, represents an approximate specification of the average interval between successive passages of any season $L_{S}$ for any planet in terms of its $\mathrm{L}_{\mathrm{SP}}$ and other orbit parameters. On the Earth, for example, where $\mathrm{e} \approx 0.0167, \mathrm{~L}_{\mathrm{SP}} \approx 283^{\circ}, \mathrm{n}_{\mathrm{sp}} \approx 4.7 \times 10^{-5} \%$, and by Newcomb's formula the tropical year is currently $\tau_{\text {trop }}=$ $(86400 / 86401.85) \times 365.25 \approx 365.2422^{d}$, the average period between successive occurrences of the vernal equinox $\left(\mathrm{L}_{\mathrm{S}}=0^{\circ}\right)$ is more nearly $\left[1+\left(2 \mathrm{e} \mathrm{n}_{\mathrm{sp}} \tau_{\text {trop }} / 360^{\circ}\right) \cos \left(\mathrm{L}_{\mathrm{SP}}\right)\right] \tau_{\text {trop }} \approx 365.2423^{\mathrm{d}}$, while the period for the northern winter solstice is $\left[1+\left(2 \mathrm{e} \mathrm{n}_{\mathrm{sp}} \tau_{\text {trop }} / 360^{\circ}\right) \cos \left(270^{\circ}-\mathrm{L}_{\mathrm{SP}}\right)\right] \tau_{\text {trop }} \approx$ $365.2428^{\mathrm{d}}$. (More precisely, the formulas given by Meeus for the Earth's four seasons imply a mean repetition interval of $365.242374^{\mathrm{d}}$ for the March equinox in the current epoch and $365.242740^{\mathrm{d}}$ for the December solstice.)

Also of critical importance to both planetary climate studies and the design of spacecraft power systems is the true solar distance. This may be efficiently 
evaluated in terms of the mean anomaly as

$$
\begin{aligned}
r / a=1 & +\frac{1}{2} e^{2}-\left(e-\frac{3}{8} e^{3}\right) \cos M-\left(\frac{1}{2} e^{2}-\frac{1}{3} e^{4}\right) \cos 2 M \\
& -\frac{3}{8} e^{3} \cos 3 M-\frac{1}{3} e^{4} \cos 4 M+O\left(e^{5}\right) .
\end{aligned}
$$

The heliocentric orbital longitude of a planet is measured from the Earth equinox along the ecliptic to the node and then along the orbit as $v+\bar{\omega}$, where $\bar{\omega}$ is the longitude of perihelion. The ecliptic longitude differs from the orbital longitude by a small quantity, known as the "reduction to the ecliptic," approximately given as $R \approx-\left(\tan ^{2} \mathrm{i} / 2\right) \cdot \sin [2(\nu+\sigma-\Omega)]$, where $\mathrm{i}$ is the orbital inclination and $\Omega$ the longitude of the orbital ascending node. (For Mars, $\mathrm{i} \approx 1^{\circ} .85$ and $\mathrm{R}$ is everywhere less than $0^{\circ} .015$.) Then with $\mathrm{v}=$ $\mathrm{L}_{\mathrm{S}}-\mathrm{L}_{\mathrm{SP}}-\mathrm{n}_{\mathrm{SP}}\left(\mathrm{t}-\mathrm{t}_{0}\right)$,

$$
\begin{gathered}
\mathrm{l}_{\text {eclip }} \approx \mathrm{L}_{\mathrm{S}}+\left[\varpi_{0}-\mathrm{L}_{\mathrm{SP}}\left(\mathrm{t}_{0}\right)\right]+\left(\mathrm{n}_{\varpi}-\mathrm{n}_{\mathrm{SP}}\right)\left(\mathrm{t}-\mathrm{t}_{0}\right) \\
-\left(\tan ^{2} \mathrm{i} / 2\right) \cdot \sin \left[2\left(\mathrm{~L}_{\mathrm{S}}-\mathrm{L}_{\mathrm{SP}}+\bar{\varpi}_{0}-\Omega_{0}\right)\right],
\end{gathered}
$$

where $\varpi_{0}$ denotes the longitude of perihelion evaluated at $t=t_{0}$ and $n_{\varpi} \equiv n_{\bar{\varpi}} / d t$ its time-linear rate of change. Specific applications of this formula or comparisons with an accurate computational ephermeris must take account of the intended frame of reference for the ecliptic and equinox as this affects the secular rates for $\bar{\omega}$ and $\Omega$. The Astronomical Almanac (1985-2000), for example, tabulates the heliocentric positions of the planets in reference to the mean equinox and ecliptic of date, as convenient for Earth-based obsrvations, while the Multiyear Interactive Computer Almanac (U.S. Naval Observatory, 1998) calculates heliocentric positions in reference to the ecliptic and equinox of J2000.0, as might be preferred for spaceflight mission analysis. For the Earth, (11) reduces to $\mathrm{l}_{\text {eclip }}=\mathrm{L}_{\mathrm{S}}-180^{\circ}$. 


\section{The Pathfinder Pole Vector}

The starting point for the new calculation of the Mars solar seasons is the planet's pole vector. Table 1 provides a comparison of the IAU definition (Davies et al., 1996), as adopted by the Astronomical Almanac, with the new results of Folkner et al. (1997), based on a combination of Pathfinder and Viking Lander radio tracking data. $\alpha_{0}$ and $\delta_{0}$ denote the right ascension and declination of the Mars pole on the celestial sphere with respect to the J2000 equinox and ecliptic, while $\alpha_{1}$ and $\delta_{1}$ (in the notation of the Astronomical Almanac) denote the transformation of these coordinates to their reference with respect to the mean equinox and ecliptic of date, which we have calculated by standard reduction methods (e.g. Meeus, 1991; Hohenkerk et al., 1992) using the Earth precession constants given by Simon et al. (1994). The derived time-linear formulas for $\alpha_{1}$ and $\delta_{1}$ corresponding to the IAU definition are in perfect agreement with the numbers given each year in the Astronomical Almanac (1985-2000, p.E87), to within the tabulated accuracy. $\lambda_{1}$ and $\beta_{1}$ denote the corresponding ecliptic longitude and latitude, again with repect to the mean equinox and ecliptic of date. The (centennial) time-linear forms for these correspond to their first-order evaluated series expansions about the J2000 epoch. Since the results vary from the exactly evaluated coordinates, as transformed from the tabulated expressions for $\alpha_{0}$ and $\delta_{0}$, by as much as $0^{\circ} .0003$

Table 1. Time-linear coordinate representations of the Mars pole vector, for $\mathrm{T} \equiv(\mathrm{JD}-2451545) / 36525$, the time in Julian centuries post-J 2000 .

\section{IAU (Davies et al., 1996) Pathfinder (Folkner et al.,1997)}

Evaluated r.a. and $\alpha_{0}=317^{\circ} .681-0^{\circ} .108 \mathrm{~T} \quad \alpha_{0}=317^{\circ} .68143-0^{\circ} .1061 \mathrm{~T}$ dec, J2000 Eqnx $\quad \delta_{0}=52^{\circ} .886-0^{\circ} .061 \mathrm{~T} \quad \delta_{0}=52^{\circ} .88650-0^{\circ} .0609 \mathrm{~T}$
r.a. and dec for
$\alpha_{1}=317^{\circ} .681+0^{\circ} .678 \mathrm{~T}$
$\alpha_{1}=317^{\circ} .68143+0^{\circ} .6798 \mathrm{~T}$
Mean Eqnx of Date
$\delta_{1}=52^{\circ} .886+0^{\circ} .351 \mathrm{~T}$
$\delta_{1}=52^{\circ} .88650+0^{\circ} .3508 \mathrm{~T}$
Ecliptic lon-lat
$\lambda_{1}=352^{\circ} .906+1^{\circ} .173 \mathrm{~T}$
$\lambda_{1}=352^{\circ} .9076+1^{\circ} .1747 \mathrm{~T}$
Mean Eqnx of Date
$\beta_{1}=63^{\circ} .282-0^{\circ} .004 \mathrm{~T}$
$\beta_{1}=63^{\circ} .2820-0^{\circ} .0046 \mathrm{~T}$ 
for $\lambda_{1}$ and $0^{\circ} .00004$ for $\beta_{1}$ for $T= \pm 1$ (Julian century), it seems sensible to round these as given to the nearest ten thousoundth degree. Our derived forms for $\lambda_{1}$ and $\beta_{1}$ corresponding to the IAU Mars pole vector agree, however, to within $0^{\circ} .0005$ of their evaluations by Meeus (1991). Although the difference of the new pole vector, as determined from Pathfinder radio tracking, from the IAU definition is small, it turns out to be significant for the discrimination of the Fictitious Mean Sun (or $\mathrm{L}_{\mathrm{s}}$ ) to the nearest $0^{\circ} .001$, as immediately apparent from a comparison of the corresponding ecliptic longitudes. At the same time, the more than factor-ten improvement in the accuracy of the Mars pole vector from the recent analysis by Folkner et al. (1997) assures the feasibility of a new evaluation of the Mars FMS to within $0^{\circ} .001$.

\section{Calculation of Mars Solar Seasons}

We have evaulated the Fictitious Mean Sun at Mars as a least-squares fit to an $a b$ initio calculation of the equinox and solstice seasons $\left(\mathrm{L}_{\mathrm{S}}=0,90,180\right.$, and $270^{\circ}$ ) for each of 134 Mars orbits, based on the Pathfinder pole vector, and including solar aberration. For this purpose, the Mars heliocentric coordinates, as referred to the ecliptic and mean equinox of date, were calculated from two different truncations of the high-precision VSOP87 representation of planetary orbits, as given in conveniently coded tabulations by Bretagnon and Simon (1986) and Meeus (1991). According to the specified accuracy for truncated representations of the VSOP87 theory, as described by Bretagnon and Francou (1988), the first of these yields the Mars heliocentric longitude to within $0^{\circ} .0025$, while the second, employing a larger number of terms, yields a maximum error of $0^{\circ} .001$. Both are therefore entirely adequate to the derivation of fitted mean quantities to within a thousandth degree. (Our evaluated fits to the Martian FMS for different intervals and with the inclusion of quadratic time dependence suggest that any higher precision would be critically contingenet upon the adopted integration span and assumed polynomial form.) VSOP87 appears to be the most sophisticated and accurate planetary theory for which the long-term orbital mean elements, as needed for the adopted fitting method, have been 
precisely calculated and published, along with a systematic evaluation of the primary perturbations of the other planets (Simon et al., 1994). These elements have been adjusted to the numerical DE200 ephemeris developed at the Jet Propulsion Laboratory (Standish et al., 1992), as also used by the Astronomical Almanac (1985-1999). Although the more recent DE403 numerical integration is now available, incorporating newer data for the asteroids, the differences are insignificant at the $0^{\circ} .001$ level. (A comparison of the osculating elements for DE200 and DE403 at 20year intervals between 1799 and 2100, as kindly supplied by Myles Standish, reveals no discrepancies larger than $0^{\circ} .0001$.) Our coded representations of the truncated series were thoroughly tested not only against the examples provided with their published tabulations, but also by extensive comparison with the U.S. Naval Observatory Astronomical Almanac and the associated Multiyear Interactive Computer Almanac (1998).

As intended for the calibration of the Fictitious Mean Sun at Mars, no allowance was made for the light-time appropriate for viewing from the Earth. The aberration of the Sun as seen at Mars must, however, be taken in account for the most precise reckoning of the apparent solar illumination there. In terms of the heliocentric coordinates $l$ and $b$, the aberrated longitude and latitude were calculated as $l_{a b}=1-0^{\circ} .00697 / \mathrm{r}$ and $b_{a b}=b-0^{\circ} .000225 \cos (1-\Omega) / r$, where $r$ is the heliocentric distance of Mars in A.U., also computed from the VSOP87 ephemerides, and $\Omega$ the longitude of the ascending node of the Mars orbit, again referred to the ecliptic and mean equinox of date. Then as in the method outlined on pages 271-276 of Meeus (1991), we coded a computation of the planetocentric solar declination at Mars as $\delta_{\mathrm{S}} \equiv$ $\sin ^{-1}\left[-\sin \beta_{1} \sin b_{a b}-\cos \beta_{1} \cos b_{a b} \cos \left(\lambda_{1}-1_{a b}\right)\right]$. It may be noted that $L_{S}$ cannot be precisely and infallibly calculated from these results as $L_{S} \equiv \sin ^{-1}\left(\sin \delta_{S} / \sin \varepsilon\right)$, using a mean element representation for the Mars obliquity $\varepsilon$, since as a result of small planetary perturbations the argument of the indicated inverse sine function occasionally exceeds unity, wherever the maximum $\delta_{S}$ for a particular orbit exceeds $\varepsilon_{\text {mean }}$. Instead, the instance of each vernal and autumnal equinox was identified with the passage of $\delta_{S}$ through $0^{\circ}$, and the solstices with its 
corresponding maxima and minima. The implied minimax search through the evaluated declinations can be accurately accomplished for a hundred orbits in only a few hours with a standard desktop computer.

The Modified Julian Date (MJD $\equiv \mathrm{JD}$ - 2400000.5) for each Mars equinox and solstice passage as calculated with the Pathfinder pole vector over 135 orbits for the years 1874-2127 are tabulated in the appendix, along with the dates for each perihelion passage. Although they serve as the basis for our fitted evaluation of the Fictitious Mean Sun it should be emphasized that the given seasonal dates have been calculated from the truncated VSOP87 ephemeris without any reliance upon mean orbital elements, except for the very weak dependence of the aberration correction on the longitude of the ascending node.

\section{Planetary Perturbations and the Meaning of "Mean"}

Since 1984, the numerical integration of the equations of motion for the $\mathrm{N}$-body interactions of the numerous separately tracked solar system objects has replaced the former calculation of ephemerides from planetary theories of perturbed mean elements, as tabulated in The Astronomical Almanac. As remarked by Seidelmann and Fukushima (1992), "a geometric mean longitude of the Sun does not exist for modern ephemerides based on a numerical integration." Mean element representations for the heliocentric motion of the Earth and other planets continue to serve several important applications, however, including observational scheduling, telescope pointing, and spaceflight mission planning.

It should be recognized that no "mean" quantity such as the orbital mean anomaly or the FMS has any precise meaning apart from a specification of its assumed fitting interval, functional formulation, and reference epoch. As a practical matter, it seems appropriate wherever possible to estimate the desired mean quantity over a fitting interval no smaller than the temporal span of its intended application. If one or more short-term oscillations are apparent, it may be desirable to extend the fit at least as far as their complete cycles of variation. For some purposes, however, it may also be desirable to restrict the span of the 
fit to sufficiently short intervals such that the long-term nonlinear variation of the derived mean quantity represents only a small or negligible correction to its otherwise uniform rate of change.

Simon et al. (1994) of the Bureau de Longitudes present a high precision tabulation of the classical mean orbital elements of the planets as defined for a span of $\pm 6000 \mathrm{yr}$, including the third, fourth, and fifth order time dependence of the longitudes, referenced both to the fixed mean dynamical equinox and ecliptic of J2000 and to the mean dynamical equinox and ecliptic of date. Their listed classical elements for the fixed J2000 equinox and ecliptic of Mars are the same as those previously reported by Kieffer et al. (1992), as attributed there to the work of Bretagnon. The included short trigonometric series representation by Simon et al. (1994) of the primary perturbations of the Mars orbital longitude by the other planets provides a useful context for the considered definition of the appropriate limits to a determination of the planet's FMS. Figure 2 shows a plot of the indicated perturbations over three different time spans: $\pm 10, \pm 100$, and \pm 1000 Julian years.

The long period (1748yr) inequality in longitude associated with the mutual interaction of Mars, Earth, and Jupiter, as separately plotted for comparison with the short-term perturbations, can be approximated by its quadratic expansion about $\mathrm{J} 2000$ as LPI $\approx 0^{\circ} .0130+4.7 \times 10^{-8}(\% / \mathrm{d}) \Delta \mathrm{t}_{\mathrm{J} 2000^{-}}-5.47 \times 10^{-13}\left({ }^{\circ} / \mathrm{d}^{2}\right) \Delta \mathrm{t}^{2}{ }_{\mathrm{J} 2000}$, where $\Delta \mathrm{t}_{\mathrm{J} 2000} \equiv \mathrm{JD}^{\mathrm{TT}}-2451545.0$ represents the elapsed time in days post-J 2000 . This quadratic fit to the long period inequality, as shown by the dashed curve in Fig. 2 , is accurate to within $0^{\circ} .0001$ over $\pm 125 \mathrm{yr}$. The expression given by Simon et al. for the Mars mean anomaly over $\pm 6000 \mathrm{yr}$, as rounded to comparable precision, is $\approx 19^{\circ} .3728+0.52402068(\%) \Delta \mathrm{t}_{\mathrm{J} 2000}+$ $1.32 \times 10^{-13}\left(\% / \mathrm{d}^{2}\right) \Delta \mathrm{t}^{2}{ }_{\mathrm{J} 2000}$. Then as added to the quadratic approximation to the long period inequality, $\mathrm{M} \approx 19^{\circ} .3858+0.52402073(\%) \Delta \mathrm{t}_{\mathrm{J} 2000}$ $-4.2 \times 10^{-13}\left(\% / \mathrm{d}^{2}\right) \Delta \mathrm{t}^{2}{ }_{\mathrm{J} 2000}$ represents an appropriately fitted estimate to the mean anomaly of Mars for applications within \pm 100 yr of the current epoch. 

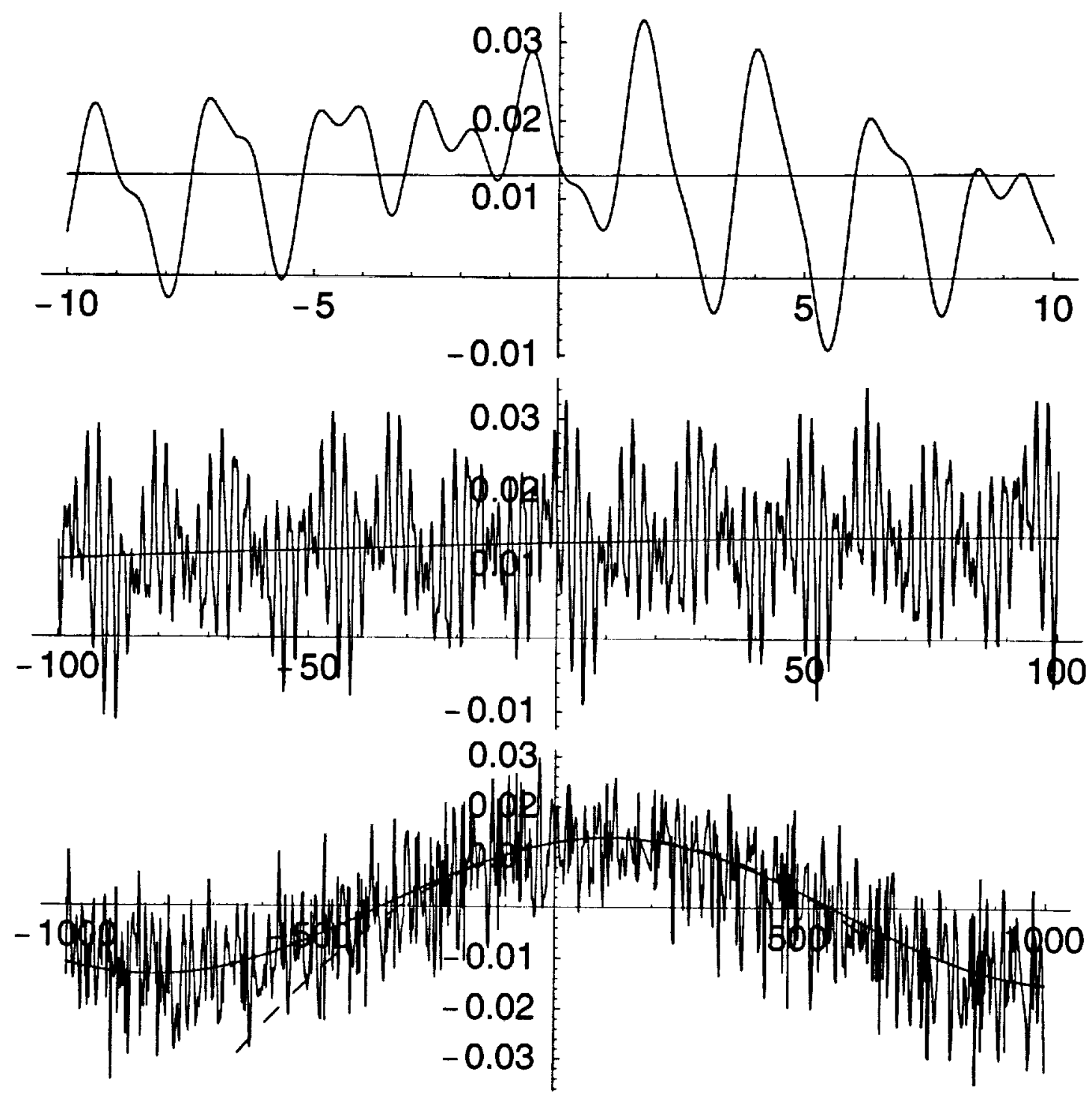

Figure 2. Mars perturbations in longitude, as evaluated for the short trigonometric series specified by Simon $e t$ al. (1994), the top panel for $\pm 10 \mathrm{yr}$, the middle panel for $\pm 100 \mathrm{yr}$, and the bottom panel for $\pm 1000 y r$. The ordinates of each plot are labeled in degrees longitude. The rapidly varying curves correspond to the superposition of seven separate perturbation terms with periods between 1 and 33years plus a long-term inequality associated with the mutual interaction of Mars, Earth, and Jupiter. The long-period (1748yr) variation in longitude is also separately plotted as the apparent smooth curve, with its quadratic fit represented by the dashed curve in the bottom panel. 
For comparison, the Mars mean anomaly given by Van Flandern and Pulkkinen (1979) for $\pm 300 \mathrm{yr}$, as translated from radians to degrees, is $19^{\circ} .3882+0.52402078(\%) \Delta \mathrm{t}_{\mathrm{J} 2000}$, within $0^{\circ} .002$ of the value inferred from the augmentation of the elements given by Simon et al. by the long period perturbation. Standish et al., present a table of the classical Keplerian elements for all nine planets in the form of the semi-major axis a, eccentricity e, inclination $\mathrm{i}$, longitude of ascending node $\Omega$, longitude of perihelion $\bar{\omega}$, and the mean longitude $L$. Their values for the Mars $L$ and $\bar{\omega}$ would imply $M=L-\bar{\omega}=$ $19^{\circ} .41248+0^{\circ} .524021165(\% / \mathrm{d}) \Delta \mathrm{t}_{\mathrm{J} 2000}$. Nearly $0^{\circ} .04$ ahead of the value derived from Simon et al. (1994), the disparity would appear to reflect a difference in the adopted fitting formulation of this representation to a Kepler orbit. (The difference represents an approximately $0^{\circ} .02$ larger value for the longitudes of both the perihelion and the ascending node with respect to the J2000 equinox and ecliptic.) It is, however, reassuring to note that the implied angular distance $L-\Omega$ along the Mars orbit between the ascending node and perihelion as given by Standish et al. is in agreement to within $0^{\circ} .00013$ of that for the mean elements given by Simon et al. (1994).

As a direct evaluation and check of an appropriate calibration of the Mars mean anomaly in application to our fit of the FMS over $\pm 126 \mathrm{yr}$, we have also performed an original quadratic fit of $M$ to our calculated dates of the perihelion passage from the truncated VSOP87 representations of the orbit. The result as inferred from the shorter truncation by Bretagnon and Simon (1986) is $\mathrm{M}=19^{\circ} .3843+0^{\circ} .52402075 \Delta \mathrm{t}_{\mathrm{J} 2000}-5.7 \times 10^{-13} \Delta \mathrm{t}_{\mathrm{J} 2000}$, as compared with $\mathrm{M}=19^{\circ} .3870+0^{\circ} .52402075 \Delta \mathrm{t}_{\mathrm{J} 2000}-5.4 \times 10^{-13} \Delta \mathrm{t}_{\mathrm{J} 2000}$ from the more accurate representation presented by Meeus (1991), again based on the full theory developed by the Bureau des Longitudes. These are each within $0^{\circ} .002$ of the long period augmentation of the elements given by Simon et al. (1994).

It is worth noting at this point that the expression (4) implies that an error or deviation in the mean anomaly $\Delta \mathrm{M}$ would result in a maximum difference for the evaluated equation of center $(\mathrm{V}-\mathrm{M})$ of $\sim 2 \mathrm{e} \sin (\mathrm{M}+\Delta \mathrm{M})<2 \mathrm{e} \bullet \Delta \mathrm{M} \approx 0.2 \Delta \mathrm{M}$ for Mars, with $e=0.0934$. An uncertainty or deviation for $M$ no larger than 
$0^{\circ} .005$, for example, therefore implies an error everywhere less than $0^{\circ} .001$. Since the fitted quadratic variation in $\mathrm{M}$ amounts to less than $0^{\circ} .001$ over the current bicentennial era (spanning the years 1900-2100), the time-linear truncation of the Mars mean anomaly expression should contribute no practical error ever larger than about $0.2 \cdot \Delta \mathrm{M} \approx 0^{\circ} .0002$ for the evaluated equation of center (4).

Table 2 specifies our adopted numerical representation of the mean orbital elements of Mars. Aside from our specially fitted value for $M$, as appropriate to its intended application over the contemporary two century interval, the other elements are taken directly from Simon et al. (1994), as simplified by their time-linear truncation and rounded specification to $\sim 1$ part in $10^{6}$ (as for an angular precision of $\sim 1 \operatorname{arcsec}$ or $0^{\circ} .0004$ ) over $\pm 200 \mathrm{yr}$.

Table 2. Adopted representation of Mars mean orbital elements. The longitude of the ascending node of the Mars orbit $\Omega$ is measured along the ecliptic from the Earth's vernal equinox, while the longitude of perihelion $\boldsymbol{\omega}$ is measured along two different planes, from the Earth's vernal equinox along the ecliptic to the orbital ascending node, and then from this node along the orbit. $\varpi, \Omega$, and $i$ are each referenced here with respect to the fixed equinox and ecliptic of J2000.

\section{Element Symbol Numerical Representation (J2000)}

Semi-major axis a

(Mean solar dist)

Eccentricity

Mean anomaly

Longitude of ascending node

Longitude of perihelion

Inclination

e

$\bar{\omega}$
M

$\Omega$

$$
336^{\circ} .0602+1.215 \times 10^{-5}{ }^{\circ} \mathrm{d}^{-1} \Delta \mathrm{t}_{\mathrm{J} 2000}
$$

$1^{\circ} .8497-2.23 \times 10^{-7}{ }^{\circ} \mathrm{d}^{-1} \Delta \mathrm{t}_{\mathrm{J} 2000}$ 


\section{The Fitted Mars FMS and Related Parameters}

Using $\mathrm{e}$ and $\mathrm{M}$ as given in Table 2 for the evaluation of the equation of center (4), and the instant of $\mathrm{L}_{\mathrm{S}}=0,90,180$, and $270^{\circ}$ evaluted as described above from two different high-precision trucations of the VSOP87 theory for each of 134 Mars orbits centered about J2000, we have constructed a quadratic fit to the Fictitious Mean Sun angle defined by equation (2). The result,

$$
\alpha_{\mathrm{FMS}}=270^{\circ} .3863+0.52403840^{\circ} \mathrm{d}^{-1} \Delta \mathrm{t}_{\mathrm{J} 2000}-4 \times 10^{-13}{ }^{\circ} \mathrm{d}^{-2} \Delta \mathrm{t}_{\mathrm{J} 2000}^{2}
$$

is very nearly the same to the indicated pecision as calculated for both representations of the VSOP87 planetary ephemeris (but with a slightly faster rate variation of $-5 \times 10^{-13} \mathrm{od}^{-1}$ for the more truncated version). It is also of some interest that the derived fit by this method is only weakly sensitive to the considered small variations among different formulations of the Mars mean anomaly. Replacing the mean anomaly formula in Table 2 with that implied by the Keplerian elements of Standish et al. (1992), for example, as for $\Delta \mathrm{M}=0^{\circ} .0255+0.00000041^{\circ} \mathrm{d}^{-1} \Delta \mathrm{t}_{\mathrm{J} 2000}$, the difference in the fitted FMS angle is only $-0^{\circ} .0004-8 \times 10^{-9}{ }^{\circ} \mathrm{d}^{-1} \Delta \mathrm{t}_{\mathrm{J} 2000}-3 \times 10^{-14}{ }^{\circ} \mathrm{d}^{-2} \Delta \mathrm{t}^{2}{ }_{\mathrm{J} 2000}$. Small $\left(\sim 0^{\circ} .03\right)$ variations among different formulations of the Mars mean anomaly therefore have an essentially insignificant effect on the derived FMS at the thousandth degree level over $\pm 125 y$. The newly determined Mars pole vector by Folkner et al. (1997) is, however, of signficance to the evaluated FMS angle, yielding a value approximately $0^{\circ} .0013$ smaller than for the current IAU definition, but of very nearly the same rate of advance, as presented by Allison (1997). [As a matter of historical interest, a repetition of the FMS fit using the pole vector of Lowell and Crommellin (1905), as adopted by the Astronomical Ephemeris prior to 1968 , yields an angle over $3^{\circ}$ smaller but with the comparable rate of approximately $0.524040 \%$ d.] The included quadratic term for the new result, while of negligible importance to many applications, is slightly larger than that in Newcomb's definition of the Fictitious Mean Sun for the Earth and represents a larger contribution to an evaluation of $\mathrm{L}_{\mathrm{S}}$ with (4) than the quadratic dependence of $\mathrm{M}$, approaching $0^{\circ} .001$ over centennnial intervals. 
As recognized by Jean Meeus (1964), the Mars tropical orbit period is shortened with respect to the sidereal revolution by an amount directly related to the planet's precession rate. And a quite accurate (if largely uncredited) value of the Mars tropical orbit period was noted by R.H. Norton in 1967, as reported in the Mars Scientific Model (JPL-D-606-1) compiled by Michaux and Newburn (1972). Surán (1997) recovered a similarly accurate estimate of the Mars tropical year as $686.972577778^{d}$, deriving this from the planet's sidereal orbit period and the modeled polar precession rate of Hilton (1991). Although specified to an unrealistic precision, this evaluation is consistent to the nearest ten-thousandth day with the determination of the FMS rate in this study.

An approximate but direct corroboration of the angular placement of the new FMS calibration is afforded by the new determination of the planet's precessional "node angle" from the combined analysis of Pathfinder and Viking lander radio tracking. As analyzed by Folkner et al. (1997), the angle specifying the position and precession of the Mars equinox as measured along the planet's mean orbital plane for the 1980 epoch from the J2000 ecliptic is $\psi_{J 2000}$ $=35^{\circ} .43777$ (at JD2451545) with $\mathrm{d} \psi / \mathrm{dt}=-7^{\prime \prime} .576 / \mathrm{yr}=-5.762 \times 10^{-6 \%} / \mathrm{d}$. As projected back to 1980 Jan 1.0 (JD 2444239.5), this implies $\psi_{1980}=35^{\circ} .47986$, within $0^{\circ} .0002$ of the value previously given by Yoder and Standish (1997) for the same date (near the midpoint of the Viking lander data record). Now the angular displacement of the dynamical mean sun from the planet's vernal equinox corresponds to the sum of the argument of the perihelion $(\omega-\Omega)$ and the orbital mean anomaly, minus the precessional node angle. As adjusted by a mean solar aberration constant $k=0^{\circ} .0042$ for Mars, the evaluated angle for the right ascension of the Fictitious Mean Sun at the 1980 epoch is $\alpha_{\mathrm{FMS}}(1980)=$ $\mathrm{M}_{1980}+(\boldsymbol{\omega}-\Omega)_{1980}-\psi_{1980}-k=42^{\circ} .0226$, using the mean elements given in Table 2. And as projected to $\mathrm{J} 2000$ (JD2451545), with $\mathrm{n}_{\text {trop }} \Delta \mathrm{t}_{\mathrm{J} 2000}=$ $3828^{\circ} .3625 \rightarrow 228^{\circ} .3625$ (reset by subtraction of $10 \times 360^{\circ}$ ), $\alpha_{\mathrm{FMS}^{(J 2000)}} \rightarrow$ $270^{\circ} .385$. This direct estimate is in fair agreement with the fitted calibration of eqn. (12), but suffers from a somewhat sensitive dependence upon the adopted mean orbital elements. (The sensitivity of this method to the mean element 
representation could presumably be eliminated by reference to a sufficiently accurate orientation of the adopted mean orbit planes, essentially equivalent to the high-precision definition of the planet's pole vector with respect to the fixed J2000 Earth equinox and ecliptic.)

The new FMS evaluation presented here implies a Mars tropical orbit period $\tau_{\text {trop }}=360^{\circ} / \mathrm{n}_{\text {trop }}=686.97256^{\mathrm{d}}$ and by (7) and (8), $\mathrm{L}_{\mathrm{SP}}=250^{\circ} .9993$, with $\mathrm{n}_{\mathrm{SP}}=$ $1.765 \times 10^{-5 \circ} \mathrm{d}^{-1}$. Other specific estimates of either the Mars tropical orbit period or the corresponding FMS rate, as distinct from the rates for the planet's sidereal and anomalistic motions, are curiously difficult to find in the accessibly published literature. Similarly, tabulations of Mars astrometric constants have rarely included an accurate evaluation of $\mathrm{L}_{\mathrm{SP}}$, with a value of $248^{\circ}$, as adopted by Levine et al. (1977), still appearing in recent application studies (e.g. François et al., 1990; Atkinson and Gwynne, 1992; Badescu, 1998), though this appears to derive from the much older pole vector estimate of Lowell. Harvey (1982), however, presented a moderately accurate estimate of $\mathrm{L}_{\mathrm{SP}}$ for the planets (with $-109^{\circ} .0$ for Mars), as inferred from the osculating elements for the start of 1981 , refering to this angle (which he designated by $\theta$ ) as the longitude of perihelion converted to "its value in each planet's natural coordinate system."

Table 3 presents a selected outline of various Mars FMS/tropical orbit period evaluations, summarizing the history of different fitting methods and available data. The JPL definitions of the FMS appear to have excluded solar aberration and have been calibrated in application to specific Mars flight projects. Of the different studies listed, only the present work has had the benefit of the new Pathfinder pole vector, as evaluated by Folkner et al. (1997). 
Table 3. A selected history of areocentric mean solar coordinate evaluations. Although some authors have prefered to retain the full precision of their arithmetic, the angular results as given here are rounded to the nearest ten-thousandth degree, with the corresponding epochs and evaluated estimate of the implied tropical year rounded to the nearest hundred-thousandth day.

\section{Author(s)}

FMS formula

\section{[Implied] Tropical \\ $\alpha_{\text {FMS }}(\mathrm{J} 2000) \quad$ Orbit Period}

$\begin{array}{lcrr}\begin{array}{l}\text { Norton (1967) } \\ \text { in Michaux and } \\ \text { Newburn (1972) }\end{array} & - & - & \begin{array}{r}668.59216^{\circ} \\ {\left[=686.9726^{\circ}\right.}\end{array} \\ \begin{array}{l}\text { Beerer (1985) } \\ \text { in Kaplan }\end{array} & -2[3] 8^{\circ} .217+0.524041^{\circ} \mathrm{d}^{-1}\left(\mathrm{t}-\mathrm{t}_{0}\right) & {\left[270^{\circ} .397\right]} & 686.969^{\mathrm{d}} \\ & \text { for } \mathrm{t}_{0}=\mathrm{JD} 2449200.5 & & \end{array}$

(1988)

Blume (1986)

$$
\begin{gathered}
250^{\circ} .9882+0.5240427^{\circ} \mathrm{d}^{-1}\left(\mathrm{t}-\mathrm{t}_{0}\right) \\
\text { for } \mathrm{t}_{0}=\mathrm{JD} 2448760.08620
\end{gathered}
$$

$\left[270^{\circ} .4019\right]$

$686.9669^{d}$

Lee (1995)

$$
\begin{gathered}
188^{\circ} .3690+0.5240429^{\circ} \mathrm{d}^{-1}\left(\mathrm{t}-\mathrm{t}_{0}\right) \\
\text { for } \mathrm{t}_{0}=\mathrm{JD} 2450701.5
\end{gathered}
$$

$686.9667^{\mathrm{d}}$

Allison (1997)

$$
\begin{aligned}
& 270^{\circ} .39+0.5240384^{\circ} \mathrm{d}^{-1}\left(\mathrm{t}-\mathrm{t}_{0}\right) \quad 270^{\circ} .39 \\
& \text { for } \mathrm{t}_{0}=\mathrm{JD} 2451545.0
\end{aligned}
$$

$686.9725^{\mathrm{d}}$

Suran (1997)

$$
-
$$

Allison and

McEwen

(This work)

$$
\begin{aligned}
& 270^{\circ} .3863+0.52403840^{\circ} \mathrm{d}^{-1}\left(\mathrm{t}-\mathrm{t}_{0}\right) \quad 270^{\circ} .3863
\end{aligned}
$$

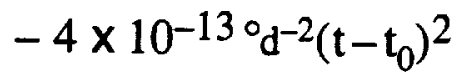

$$
\begin{aligned}
& \text { for } \mathrm{t}_{0}=\mathrm{JD} 2451545.0
\end{aligned}
$$

$686.97256^{d}$ 
The evaluated tropical year is also required for the most accurate specification of the planet's mean solar day. In the course of one solar (tropical) orbit, a planet completes one less solar rotation than the total number of sidereal rotations, so that the mean solar day or "sol" $\left(\mathrm{d}_{\mathrm{sol}}\right)$ relates to the sidereal day $\left(\mathrm{d}_{\text {sid }}\right)$ as $\tau_{\text {trop }} / \mathrm{d}_{\text {sol }} \equiv \tau_{\text {trop }} / \mathrm{d}_{\text {sid }}-1$. Equivalently,

$$
\mathrm{d}_{\text {sol }} \equiv \frac{\mathrm{d}_{\text {sid }}}{1-\mathrm{d}_{\text {sid }} / \tau_{\text {trop }}}
$$

Unfortunately, Surán made a small error in his estimate of the Mars solar day as $\mathrm{d}_{\text {sid }}+\mathrm{d}_{\text {sid }}{ }^{2} / \tau_{\text {trop }}=1.027488966^{\mathrm{d}}$, really an imperfect approximation to $\mathrm{d}_{\text {sid }}\left(1-\mathrm{d}_{\text {sid }} / \tau_{\text {trop }}\right)^{-1}$. With $\mathrm{d}_{\text {sid }}=360^{\circ} /\left(350^{\circ} .89198521 / \mathrm{d}\right)=1.02595674787^{\mathrm{d}}$, as determined by Folkner et al. (1997), the correct value for $d_{\text {sol }}=1.02749125^{\mathrm{d}}$ implies a Mars tropical year of 668.5921 Mars solar days or "sols."

Our calculation of the maximum solar declination for each orbit also affords an evaluated fit to the current Mars mean obliquity of date as $\varepsilon=$ $25^{\circ} .1919+0^{\circ} .0126 \mathrm{~T}$, where again $\mathrm{T}$ is measured in Julian centures post-J 2000 . This implies that in 1980 (at $\mathrm{T}=-0.2$ ), $\varepsilon(1980) \approx 25^{\circ} .1894$, in good agreement with the value of $25^{\circ} .189417$ established by Folkner et al. (1997) for their analysis of the Mars precession constants, taking as a reference a fixed 1980 Mars mean orbit and equinox. (The inferred centennial rate for the mean obliquity of date should not be confused with the $\mathrm{d} \varepsilon / \mathrm{dt} \approx 1 \mathrm{mas} / \mathrm{yr}$ reported in their paper, which as they note is consistent to within observational limits with a vanishing value for a fixed orbital reference plane.) The derived centennnial rate is in fair agreement with the pre-Viking value of $\mathrm{d} \varepsilon / \mathrm{dt}=0^{\circ} .01220+0^{\circ} .00006 \mathrm{~T}$ given by Sturms (1980), as reported in Michaux and Newburn (1972).

Table 4 presents a summary of the derived and related Mars orbital/ rotational parameters, including for comparison the corresponding values for the Earth. There is as yet some uncertainty in the precise location of the Mars prime meridian, as apparent in the ongoing revision of its (angle $\mathrm{W}$ ) definition by Davies et al. $(1994,1999)$ and the indicated value for $\mathrm{V}_{\mathrm{m}}=180^{\circ}+\phi$, measured as in Michael (1979) and Folkner et al. (1997) with respect to the planet's equinox, represents a compromise between extreme values in various reports. 
Table 4. Solar orbital/rotational parameters for Mars and Earth.

Symbol

Mars

Earth

\section{Parameter \\ Mean solar distance}

Orbital eccentricity

Perihelion Date

(mean element)

Planetocentric Orbital Lon

of Perihelion adj to J2000

Rate of perihelion advance

with respect to equinox

Anomalistic Orbit Period

Sidereal Orbit Period

Sidereal Day

Solar Day

Obliquity of Eqtr

to Orbit

Prime Meridian

Hour-Angle wrt

Planet's Vern Eqnx

Tropical Orbit Period $\tau_{\text {anom }}$

e

$t_{p} \quad 1999$ Nov $25\left(12^{h}\right)$
$=$ JD 2451508.0

$\mathrm{L}_{\mathrm{SP}}$

$\mathrm{n}_{\mathrm{SP}}$

$1.765 \times 10^{-5 \circ} \mathrm{d}^{-1}$

$$
=0.64467^{\circ} / \mathrm{cy}
$$

1.52368 A.U.
$1.49598 \times 10^{8} \mathrm{~km}$

$$
\begin{gathered}
0.09340 \\
+0.000090 \mathrm{~T}
\end{gathered}
$$

$250^{\circ} .999$

$686.9957^{\mathrm{d}}$

$1.880892^{\text {Julian }} \mathrm{Yr}_{\mathrm{r}}$

$668.6146^{\text {sol }}$

$\tau_{\text {sid }}$

$686.9798^{d}$

$1.880848^{\text {Julian }} \mathrm{Yr}$

$668.5992^{\text {sol }}$

$\tau_{\text {trop }}$

$686.9726^{\mathrm{d}}$

$1.880828^{\text {Julian }} \mathrm{Yr}$

$668.5921^{\mathrm{sol}}$

$\mathrm{d}_{\text {sid }} \quad 24^{\mathrm{h}} 37^{\mathrm{m}_{22.663} \mathrm{~s}}$

$=1.025956748^{\mathrm{d}}$

$\begin{array}{ll}\mathrm{d}_{\text {sol }} & 24^{\mathrm{h}_{39} \mathrm{~m}_{35.244^{\mathrm{s}}}} \\ = & 1.027491251^{\mathrm{d}}\end{array}$

$\varepsilon$

$25^{\circ} .1919$

$+0^{\circ} .0126 \mathrm{~T}$

$\begin{array}{ll}\mathrm{V}_{\mathrm{m}} & 313^{\circ} .5\left( \pm 0^{\circ} .15 ! ?\right)+ \\ & 350^{\circ} .8919851 \Delta \mathrm{t}_{\mathrm{J} 2000}\end{array}$
1.00000 A.U.

$2.27939 \times 10^{8} \mathrm{~km}$

$$
\begin{gathered}
0.01671 \\
-0.000042 \mathrm{~T}
\end{gathered}
$$

2000 Jan $4\left(0^{\mathrm{h}}\right)$

$=\mathrm{JD} 2451547.5$

$282^{\circ} .932$

$4.695 \times 10^{-5 \circ} \mathrm{d}^{-1}$

$=1.715 \% \mathrm{cy}$

$365.2596^{\mathrm{d}}$

$1.000026^{\mathrm{Jyr}}$

$365.2564^{\mathrm{d}}$

$1.000018^{\mathrm{Jyr}}$

$365.2422^{\mathrm{d}}$

$0.999979 \mathrm{Jyr}$

$23^{\mathrm{h}_{56}} \mathrm{~m}_{04.09054^{\mathrm{s}}}$

$=0.997269566^{\mathrm{d}}$

$24^{\mathrm{h}_{00} \mathrm{~m}_{00} \mathrm{~s}}$

$=1.00000000^{\mathrm{d}}$

$$
\begin{gathered}
23^{\circ} .4393 \\
-0^{\circ} .0130 \mathrm{~T}
\end{gathered}
$$

$100^{\circ} .460+$
$360^{\circ} .9856474 \Delta \mathrm{t}_{\mathrm{J} 2000}$ 
By a numerical evaluation of (9) with the parameters specified in Table 4, the Modified Julian Date of season $\mathrm{L}_{S}$ within the $\mathrm{n}^{\text {th }}$ orbit of Mars since the epoch 1874.0 may be estimated for any subsequent date as

$$
\begin{gathered}
\operatorname{MJD}\left(\mathrm{n}, \mathrm{L}_{\mathrm{S}}\right)=51507.5+1.90826\left(\mathrm{~L}_{\mathrm{S}}-251^{\circ}\right)-20.42 \sin \left(\mathrm{L}_{\mathrm{S}}-251^{\circ}\right) \\
+0.72 \sin \left[2\left(\mathrm{~L}_{\mathrm{S}}-251^{\circ}\right)\right]+\left\{686.9726+0.0043 \cos \left(\mathrm{L}_{\mathrm{S}}-251^{\circ}\right)\right. \\
\left.-0.0003 \cos \left[2\left(\mathrm{~L}_{\mathrm{S}}-251^{\circ}\right)\right]\right\} \text { IntegerPart }[\mathrm{n}-66]
\end{gathered}
$$

The last term in the brackets multiplying the orbit index $n-66$ indicates the variation in the average repetition interval for a Mars season $L_{S}$, as plotted in Figure 3. It may be readily verified that the indicated variation for the repetition of the equinoxes and solstices is consistent with the repetition of seasonal dates listed in the appendix. A computer-encoded rendition of this equation, looped through $\mathrm{Ls}=0,90,180$, and $270^{\circ}$ and $n=0,1,2, \ldots, 134$, efficiently recovers the dates derived from the VSOP87 representation, as tabulated in the appendix, to within a maximum error of $0.1^{\mathrm{d}}$.

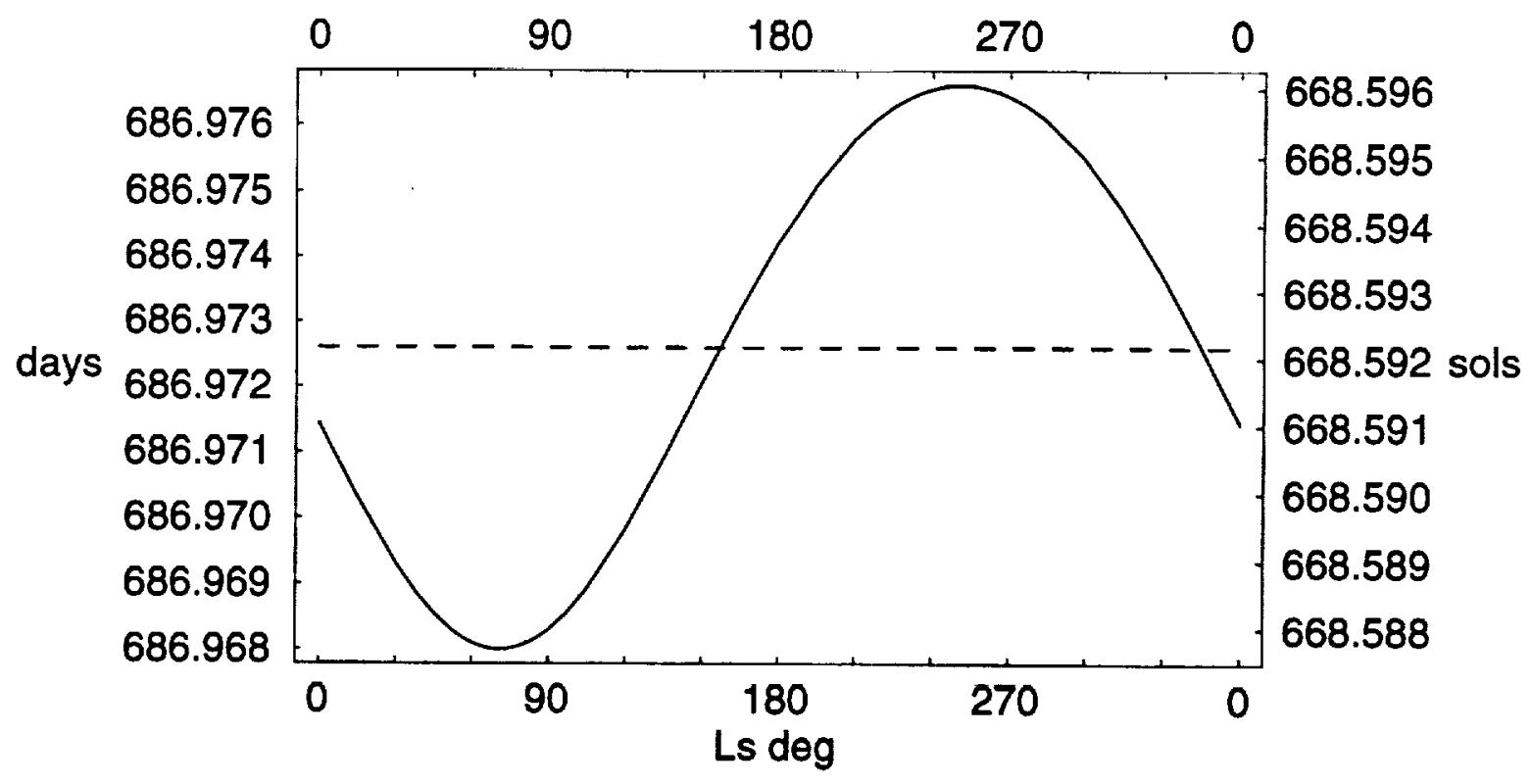

Figure 3. Variation of the Mars solar seasonal year, shown as the mean interval in days and sols for the repetition of a given Ls. The dashed horizontal line represents the average tropical year, $686.9726^{\mathrm{d}}$ or $668.5922^{\mathrm{sol}}$, corresponding to the interval for the repetition of the Fictitious Mean Sun angle. 


\section{Mars Solar Timing Algorithms}

The following algorithm, including the primary peruturbations of Jupiter, Earth, and Venus, affords a quick calculation of Mars solar coordinates. As referenced to the elapsed time in days from the $\mathrm{J} 2000$ epoch (2000 Jan $1.5^{\mathrm{TT}}$ ) with

$$
\Delta \mathrm{t}_{\mathrm{J} 2000} \equiv\left(\mathrm{JD}^{\mathrm{TT}}-2451545.0\right)
$$

(where JD is the Julian Date in Terrestrial Time, offset from Universal Coordinated Time as prescribed below), the mean anomaly, Fictitious Mean Sun angle, and the sum of the angular perturbations in longitude are given as

$$
\begin{gathered}
\mathrm{M}=19^{\circ} .3870+0^{\circ} .52402075 \Delta \mathrm{t}_{\mathrm{J} 2000} \\
\alpha_{\mathrm{FMS}}=270^{\circ} .3863+0^{\circ} .52403840 \Delta \mathrm{t}_{\mathrm{J} 2000}-4 \times 10^{-13} \Delta \mathrm{t}^{2}{ }_{\mathrm{J} 2000} \\
\mathrm{PBS}=\sum_{\mathrm{i}=1}^{7} \mathrm{~A}_{\mathrm{i}} \cos \left[0^{\circ} .985626 \Delta \mathrm{t}_{\mathrm{J} 2000} / \tau_{\mathrm{i}}+\phi_{\mathrm{i}}\right]
\end{gathered}
$$

where $A_{i}, \tau_{i}$, and $\phi_{i}$ denote the amplitude, period, and phase of the planetary perturabions given in Table 5. Then the areocentric solar longitude and equation

Table 5. Primary short-term perturbations for the areocentric solar longitude, represented in the form $A_{i} \cos \left[\left(360^{\circ} / 365.25\right) \Delta t_{\mathrm{J} 2000} / \tau_{\mathrm{i}}+\phi_{\mathrm{i}}\right]$, as approximated by a moderate-precision transformation of the associated sine and cosine terms given by Simon et al. (1994) and plotted in Fig.2. The right-most column identifies the planetary commensurability responsible for each perturbation, in terms of the indicated differences of integral multiples of mean motion rates for Jupiter $\left(\lambda_{\mathrm{J}}\right)$, Mars $\left(\lambda_{M}\right)$, Earth $\left(\lambda_{E}\right)$, and Venus $\left(\lambda_{V}\right)$.

\begin{tabular}{ccccc}
\hline $\mathrm{i}$ & $\mathrm{A}_{\mathrm{i}}(\mathrm{deg})$ & $\tau_{\mathrm{i}}(\mathrm{Jyr})$ & $\phi_{\mathrm{i}}(\mathrm{deg})$ & $\begin{array}{c}\text { Planetary } \\
\text { Commensurability }\end{array}$ \\
\hline 1 & 0.0071 & 2.2353 & 49.409 & $\left(\lambda_{\mathrm{M}}-\lambda_{\mathrm{J}}\right)^{-1}$ \\
2 & 0.0057 & 2.7543 & 168.173 & $\left(\lambda_{\mathrm{M}}-2 \lambda_{\mathrm{J}}\right)^{-1}$ \\
3 & 0.0039 & 1.1177 & 191.837 & $\left(2 \lambda_{\mathrm{M}}-2 \lambda_{\mathrm{J}}\right)^{-1}$ \\
4 & 0.0037 & 15.7866 & 21.736 & $\left(2 \lambda_{\mathrm{M}}-\lambda_{\mathrm{E}}\right)^{-1}$ \\
5 & 0.0021 & 2.1354 & 15.704 & $\left(\lambda_{\mathrm{E}}-\lambda_{\mathrm{M}}\right)^{-1}$ \\
6 & 0.0020 & 2.4694 & 95.528 & $\left(2 \lambda_{\mathrm{E}}-3 \lambda_{\mathrm{M}}\right)^{-1}$ \\
7 & 0.0018 & 32.8493 & 49.095 & $\left(\lambda_{\mathrm{V}}-3 \lambda_{\mathrm{M}}\right)^{-1}$ \\
\hline
\end{tabular}


of time are given as

$$
\begin{aligned}
\mathrm{L}_{\mathrm{S}}= & \alpha_{\mathrm{FMS}}+\left(10^{\circ} .691+3^{\circ} \times 10^{-7} \Delta \mathrm{t}_{\mathrm{J} 2000}\right) \sin \mathrm{M}+0^{\circ} .623 \sin 2 \mathrm{M} \\
& +0^{\circ} .050 \sin 3 \mathrm{M}+0^{\circ} .005 \sin 4 \mathrm{M}+0^{\circ} .0005 \sin 5 \mathrm{M}+\mathrm{PBS}
\end{aligned}
$$

and

$$
\begin{aligned}
\text { EOT }= & 2^{\circ} .861 \sin 2 \mathrm{~L}_{\mathrm{S}}-0^{\circ} .071 \sin 4 \mathrm{~L}_{\mathrm{S}}+0^{\circ} .002 \sin 6 \mathrm{~L}_{\mathrm{S}} \\
& -\left\{\left(10^{\circ} .691+3^{\circ} \times 10^{-7} \Delta \mathrm{t}_{\mathrm{J} 2000}\right) \sin \mathrm{M}+0^{\circ} .623 \sin 2 \mathrm{M}\right. \\
& \left.+0^{\circ} .050 \sin 3 \mathrm{M}+0^{\circ} .005 \sin 4 \mathrm{M}+0^{\circ} .0005 \sin 5 \mathrm{M}+\mathrm{PBS}\right\}
\end{aligned}
$$

The given EOT expression is of a similar form to that presented by Yallop and Hohenkerk (1992) for the Earth-Solar ephemeris. Although the apparent redundancy of (19) and (20) can be avoided with an appropriate encoding of substituted variables, the given form for the EOT assures a result in the plus or minus small angle range for any multiple $360^{\circ}$ addition/subtraction to $\mathrm{L}_{\mathrm{S}}$. For most purposes, however, it is convenient to reset the areocentric longitude to within the $0-360^{\circ}$ range, as for example

$$
\mathrm{L}_{\mathrm{S}} \rightarrow \text { FractionalPart }\left[1+\text { FractionalPart }\left[\frac{\mathrm{LS}}{360^{\circ}}\right] 360^{\circ}\right.
$$

The Mean Solar Time on the Mars prime meridian, given as $\left(V_{m}-\alpha_{F M S}\right) \times\left(24 h / 360^{\circ}\right)+12^{h}$, may be evaluated as

$$
\text { MST }=24^{\mathrm{h}} \times \text { FractionalPart }\left[\frac{\mathrm{JD}-2451549.5}{1.02749125}+44796.0-\mathrm{k}\right] \text {, }
$$

with $\mathrm{k} \sim 0.0006 \pm 0.0004$ an as yet imprecisely determined adjustment accounting for the true position of crater Airy -0 .

As defined, consistent with the terrestrial convention for Mean Solar Time, JD2451549.5 (2000 Jan06 00:00) corresponds to a near coincidence of the terrestrial Greenwich mean solar midnight and the Martian mean solar (prime meridian) midnight. The addition of the integer number 44796 assures a positive result for the indicated fractional part in (21) for any date since JD2405522 (1873 Dec29.5). More significantly, the interval between these dates, $(2451549.5-2405522.0)^{\mathrm{d}}=46027.5^{\mathrm{d}}=44796.002^{\text {sol }}$, represents not only a 
near half-day/sol commensurability, but also a very near orbit-period commensurability of JY $2000.012-J Y 1873.996=126.016^{\mathrm{JY}}=67.0005$ Mars tropical revolutions (approximately 59 synodic periods).

The Local True Solar Time is then given as

$$
\mathrm{LTST}=\mathrm{MST}-\Lambda_{\mathrm{W}}\left(24 \mathrm{hr} / 360^{\circ}\right)+\mathrm{EOT} \bullet\left(\mathrm{hr} / 15^{\circ}\right)
$$

where $\Lambda_{\mathrm{W}}$ denotes the west longitude (measured westward from the prime meridian according to the planetary cartographic convention in the range $0-360^{\circ}$ ). Each hour-angle can be reset to $0-24^{\mathrm{hr}}$ as, for example,

$$
\text { LTST } \rightarrow \text { FractionalPart }\left[1+\text { FractionalPart }\left[\frac{\text { LTST }}{24}\right]\right] 24^{\mathrm{hr}},
$$

and then converted as desired from fractional hours to minutes (and seconds).

The Mars heliocentric distance and ecliptic longitude are numerically given from eqns.(10) and (11) as

$$
\begin{aligned}
\mathrm{r} / \mathrm{a} \approx 1.5236(1.00436-0.09309 \cos \mathrm{M} \\
-0.00436 \cos 2 \mathrm{M}-0.00031 \cos 3 \mathrm{M}) \mathrm{A} . \mathrm{U} .
\end{aligned}
$$

and

$$
\mathrm{l}_{2000} \approx \mathrm{L}_{\mathrm{S}}+85^{\circ} .061-0^{\circ} .015 \sin \left(2 \mathrm{~L}_{\mathrm{S}}+71^{\circ}\right)-5^{\circ} .5 \times 10^{-6} \Delta \mathrm{t}_{\mathrm{J} 2000}
$$

For the most precise calculation of the solar hour-angle, the Julian Date (JD) and the elapsed time $\Delta \mathrm{t}_{\mathrm{J} 2000}$ as defined by (15) for the given timing formulae should be referred to Terrestrial (Dynamical) Time (TT), or Ephemeris Time (ET) as used prior to 1984 , measured in continuous even increments of SI seconds, currently defined with reference to atomic clocks (with $\mathrm{TT}=\mathrm{TAI}+32.184^{\mathrm{sec}}$ ). An approximate conversion of Universal (Coordinated) Time (UTC) or the former Greenwich Mean Time (GMT) to Terrestrial Time may be prescribed as

$$
\mathrm{TT}=\mathrm{UTC}+64.184^{\mathrm{sec}}+95^{\mathrm{sec}} \mathrm{T}+35^{\mathrm{sec}} \mathrm{T}^{2},
$$


where $\mathrm{T} \equiv(\mathrm{JD}-2451545) / 36525=(\mathrm{Jyr}-2000) / 100$ is the elapsed time in Julian centuries from J2000. This is accurate to within $5 \mathrm{sec}$ for the years 1950-2000, and to within $11 \mathrm{sec}$ for the two-century interval 1800-2000. Although a precise UTC conversion cannot be predicted several years in advance, the formula agrees to within $1.4 \mathrm{sec}$ of extrapolated values for the years 1997-2003, spanning the operation of the Mars Pathfinder, Global Surveyor, and Climate Orbiter missions, and is exact for $\mathrm{J} 2000$, at $\mathrm{T}=0$. (UTC is itself stepped by $1 \mathrm{sec}$ increments, wherever needed at the end of June and/or December, to bring civil time keeping to within $0.9^{\mathrm{S}}$ of the exact measure of the Earth's rotation, as announced every six months by the Central Bureau of the International Earth Rotation Service at the Paris Observatory.)

Omitting the perturbations (taking PBS $=0$ ), the given $\mathrm{L}_{S}$ formula (19), as derived from Eqns.(1), (2), and (4), yields a result with a maximum error of $0^{\circ} .03$, comparable to the sum of the tabulated perturbation amplitudes. The errors in $\mathrm{L}_{S}$, as calculated by the given series expansion both with and without the primary perturbation corrections, are shown Figure 5, as evaluated by comparison with the more accurate VSOP87 ephemeris. As the lower panel suggests, approximately $95 \%$ of the values calculated with the inclusion of the seven-term perturbation correction for the equinox and solstice dates given in Table A 1 are within $0^{\circ} .005$ of the accurately computed values. The accuracy of the Ls algorithm is therefore comparable to its tabulation in the Astronomical Almanac, which differs, however in its assumption of the older IAU pole vector and the inclusion of a light-time correction. (The calculation of $\mathrm{L}_{\mathrm{S}}$ to within a maximum error of $0^{\circ} .001$ would require some 70 perturbation terms!)

Since the error in the calculated EOT (expressed in degrees) as derived by the series method is comparable to that for the Ls, the $0^{\circ} .01$ precision of the given seven-term perturbation corrections, if included, would enable the evaluation of the LTST to within $2.4^{\mathrm{S}}$. Even without the perturbation corrections, the estimated maximum error of $0^{\circ} .03$ for the short series representation of the elliptic motion over $\pm 125 \mathrm{yr}$ would imply an astrometric contribution to the LTST error never larger than $8^{\mathrm{S}}$. 


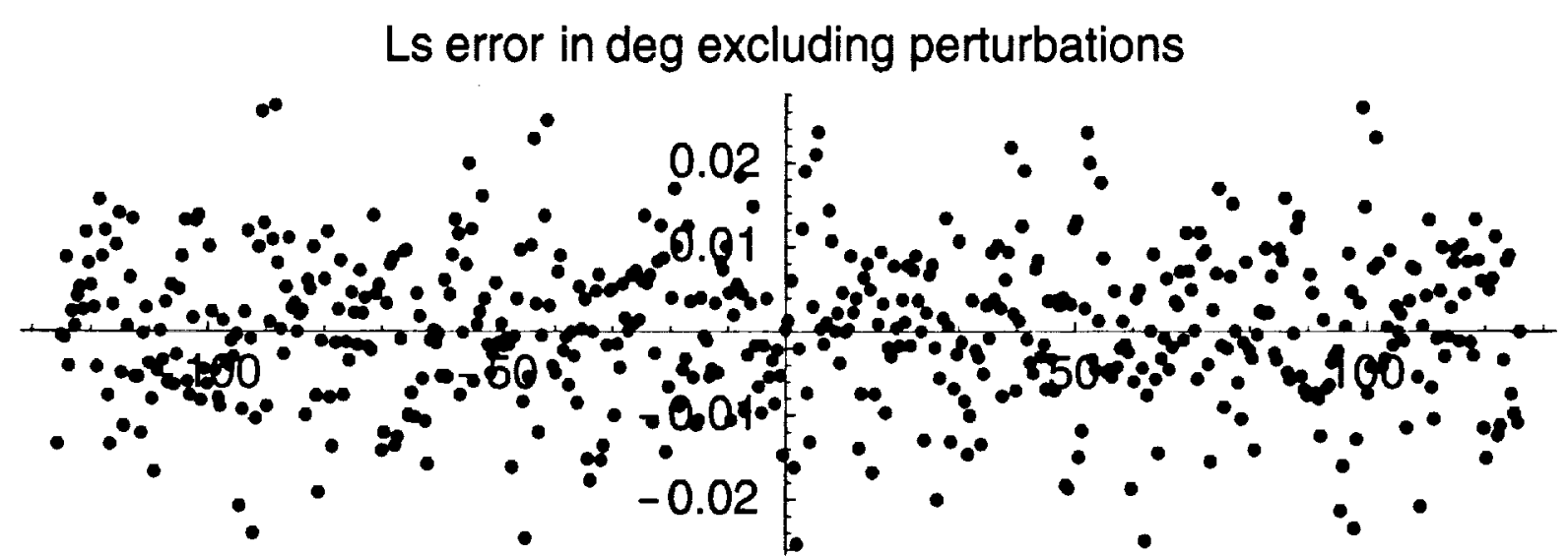

Ls error in deg with perturbation corrections

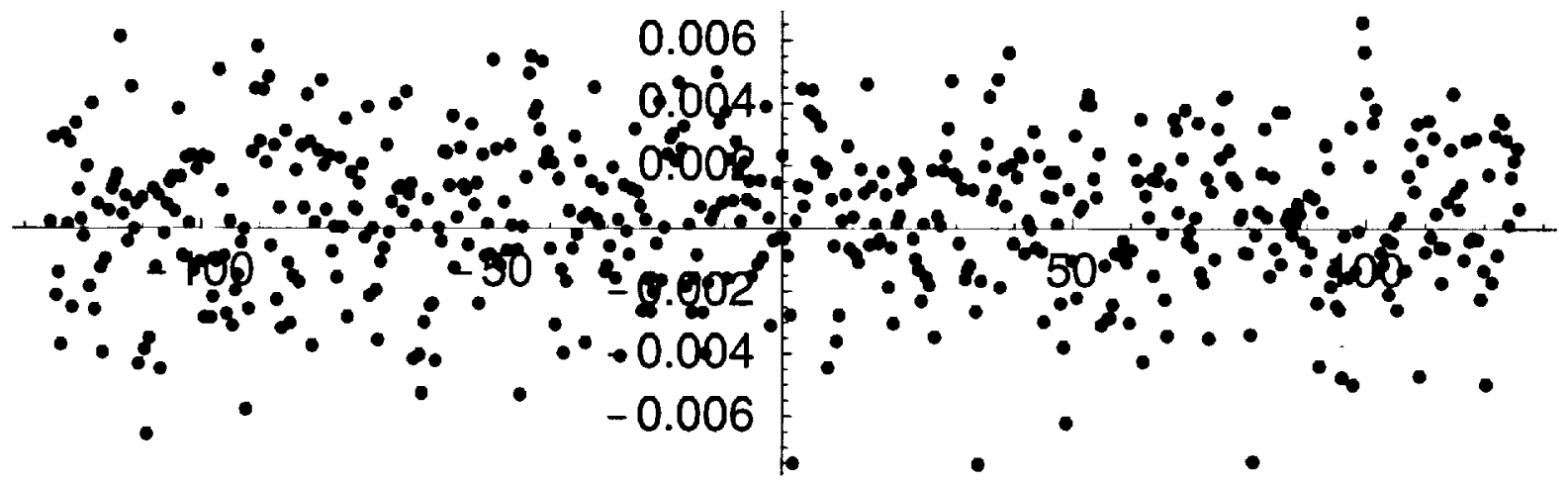

Figure 4. Errors in the Mars Ls (in deg) for each equinox and solstice over $\pm 126 \mathrm{yr}$ of $\mathrm{J} 2000$, as evaluted by the series expression (19) in comparison with the more exact calculation with the truncated VSOP87 representation, itself accurate to within $0^{\circ} .001$. The upper plot shows the errors excluding the perturbation corrections. The lower plot shows the reduced errors including the perturbations for the the 7-term cosine series with the amplitudes, phases, and periods given in Table 3, for which the maximum difference is $0^{\circ} .0075$. 


\section{Lander Solar Timing}

According to Colburn et al. (1989), the Viking "Local Lander Time" or LLT was defined so that the Sun would cross the nadir meridian at midnight on the first sol after touch-down, but otherwise took no account of the subsequent progression of the Equation of Time (EOT). Viking LLT was therefore a variant of mean solar time, offset by approximately -2.88 and $-14.69 \mathrm{hr}$ from the MST on the Mars prime meridian for Viking Landers 1 and 2, respectively. (cf. Allison, 1997.) Viking Lander "Sol numbers" were essentially reckoned from a zero starting point at each of the local true solar midnights immediately prior to their touchdowns. Time tags for the Mars Pathfinder lander were referenced with respect to the local true solar time, with elapsed sols again reckoned from the local true solar midnight preceeding its landing, but designated with a starting number "1" rather than zero. Table 6 gives the exact times for the Viking and Mars Pathfinder landings, along with estimated epochs for the immediately prior local mean midnights, as usefully referenced for the calculation of their mission sol number and local solar time.

Table 6. Mars lander chronological epochs. Times for the Viking lander touchdowns are from Snyder (1977). The time for the Mars Pathfinder landing is from Golombek et al. (1997). Note that the Julian Dates are given here in Universal Coordinated Time (UTC).

Touchdown Date/Time

Local Mean Midnight Epoch

$\mathbf{t}_{\mathrm{ME}}$ (Ref'd prior to TD)

Viking

Lander 1

Viking

Lander 2

Mars

Pathfinder
1976 Jul 20 11:53:06 UTC

= JD 2442979.99521 UTC

1976 Sep 03 22:37:50 UTC

= JD 2443025.44294 UTC

1997 Jul 04 16:56:55 UTC

= JD 2450634.20619 UTC
VL1 Sol $0.0=$

JD 2442979.319 UTC

VL2 Sol $0.0=$

JD 2443025.034 UTC

MPF (Mean) Sol $1.0=$

JD 2450634.10048 UTC 
The Viking 1 "sol number", for example, is readily evaluated as

$$
\text { VL1 Sol No. = IntegerPart[(JD UTC }-2442979.319) / 1.02749125] \text {, }
$$

and with an appropriately specified decimal fraction for the Julian Date corresponding to a particular terrestrial calendar date and time, the VL1 "Local Lander Time" (in hours) can be efficiently estimated as

VL1 LLT $=24^{\mathrm{h}} \times$ FractionalPart[(JD UTC -2442979.319$\left.) / 1.02749125\right]$.

A decimal Mars Pathfinder "sol date", as referenced to the local true solar time, may be calculated as

$$
\mathrm{MPF} \text { TSD }=\left(\mathrm{JD}^{\mathrm{UTC}}-2450634.10048\right) / 1.02749125+\mathrm{EOT} / 360^{\circ}+1,
$$

with EOT evaluated by eqn. (20) for the exact time of interest and then converted as indicated from degrees to the appropriate decimal sol fraction. The Pathfinder Local True Solar Time is then readily computed as

$$
\text { MPF LTST }=24^{\mathrm{h}} \times \text { FractionalPart[MPF TSD]. }
$$

The evaluated decimal hour angle for the landers may be converted to hours minutes and seconds, consistent with the spacecraft data records generated for these missions at the Jet Propulsion Laboratory (e.g. Golombek, 1997).

\section{Discussion}

The new calibration of the Fictitious Mean Sun at Mars presented here, as specified to $0^{\circ} .0001$, would serve to define a Martian "Mean Solar Time" (MST) consistent with the historical conventions established for terrestrial chronology to within $\left(0^{\circ} .0001 / 360^{\circ}\right) \times 86400^{\mathrm{S}}=0.024^{\mathrm{S}}$ on a $24^{\mathrm{h}} \mathrm{clock}$, once the position of the planet's prime meridian is navigated to the same precision. Of course the local true solar time (LTST), as might be preferred for the timing of scientific observations and the motorized tracking of solar power arrays on Mars, can be evaluated independently of an FMS or MST, given the local longitude and an accurate determination of the true solar right ascension. With the slow sol-to-sol variation of the equation of time, at most some $40^{\mathrm{S}}$ (cf. Allison, 1997), 
conceiveably both human and machine activities on Mars could be reckoned with respect to the variably advancing LTST, or a globally synchronized TST (e.g. an "Airy True Solar Time" on the prime meridian).

Even if the use of MST on Mars were to be entirely abandoned, however, it seems unlikely that the Permanent Read-Only-Memory (PROM) of every instrument timer and clock on the planet would in this case be equipped with a complete numerical solar-system ephemeris for the calculation of the true solar right ascension and LTST at each instant. It would be relatively easy, however, to encode the PROM of any digital clock with a solar timing algorithm of the simple form presented here, as calibrated with respect to the FMS formula. The estimated $\sim 3 \mathrm{sec}$ accuracy of the given expression for the equation of time $(\sim 8 \mathrm{sec}$ excluding the planetary perturbations) would appropriately serve the long-term stability of many quartz clocks (e.g. typically a few seconds per month).

Measurements by lander radio tracking of a very small seasonal modulation of the Mars rotation ( 2 milli-arcsec for its angular measure), as attributed to the sublimation of the polar caps, have been modeled using the orbital mean anomaly as the dependent variable (Yoder and Standish, 1997; Folkner et al., 1998). $\alpha_{\text {FMS }}$ or some redefined angle advancing at the FMS rate is likely to be a more appropriate dependent variable, however, for the measured or modeled Mars surface pressure, condensed $\mathrm{CO}_{2}$, water vapor distribution, or any other climatological field governed by the solar season. The accurately referenced FMS rate provides the most accurate representation of the solar seasonal year on Mars and, together with the planetary rotation with respect to the Mars equinox, the most accurate determination of the length of the mean solar day.

The desirability of some coherent system for the chronological reckoning of Mars solar days, logically extending the repeated sequential numbering posttouchdown for each landed spacecraft mission, is already apparent. Gangale (1997), Hartmann (1997), Suran (1997), and others have already gone so far as to propose various Mars calendar systems, inevitably involving the consideration of various social conventions as to the organization of week days and the peculiar matter of month names. For scientific purposes, however, including the 
maintenance of climatological records and spaceflight mission planning, it seems desirable to adopt some extended sol-number system for Mars analogous to the Julian Date chronology. Such a "Consecutive Mars Day" system was actually proposed by Norton (in 1967, reported in the JPL document prepared by Michaux and Newburn, 1972), as referred to a "Mars Year 1000" coincident with the Mariner 4 flyby, and calibrated with respect to the older Mars pole vector based on the early work by Lowell.

For historical utility with respect to the Earth-based atmospheric, visual mapping, and polar-cap observations of Mars (e.g. Zurek and Martin, 1993), a sequential count of sol-numbers might appropriately be started prior to the 1877 perihelic opposition. Although the initial epoch for such a system can be regarded as largely arbitrary, it might be of some computational facility to select as a convenient starting point a near coincidence of Earth-Mars mean solar midnights as defined for each of their cartographic prime meridians. Two such near-alignments are built into the mean solar timing expression given by eqn.(21). As extended in an obvious way for the representation of a "Mars SolDate" (MSD) and referring this to the Modified Julian Date $(\mathrm{MJD}=\mathrm{JD}-2400000.5)$,

$$
\mathrm{MSD}=(\mathrm{MJD}-51549.0) / 1.02749125+44796.0-\mathrm{k},
$$

where again $\mathrm{k}$ is a small $\left(\sim 0.001^{\mathrm{sol}}\right)$ timing adjustment to the inertial position of the crater Airy- 0 defining the Mars prime meridian. The interval between the implied MSD $0.0 \approx$ MJD 05521.5 (1873 Dec 29.5) and MSD $44796.0 \approx$ MJD 51549.0 (2000 Jan 6.0) represents both a half-day/sol commensurability and an orbit-period commensurability of $126^{\mathrm{JY}} \approx 67$ Mars tropical orbits. As may be readily verified with eqns. (15)-(19), MSD 0 also corresponds to a Mars Ls $\approx 277^{\circ} .1$, nearly the same as the Earth Ls $\left(277^{\circ} .9\right)$ at the same epoch, and by eqn.(26) the corresponding Mars ecliptic longitude is $2^{\circ} 27^{\prime}$. The Mean Solar Time for any subsequent Mars Sol-Date is just $24^{\mathrm{h}} \times$ FractionalPart[MSD]. With an appropriate choice of coordinates, Mars seasonal/solar timing is therefore readily calculated with a simple set of formulae. 


\section{References}

Allison, M. (1997) Accurate analytic representations of solar time and seasons on Mars with applications to the Pathfinder/Surveyor missions. Geophys. Res. Lett. 24, 1967-1970.

Astronomical Almanac (1985-2000) U.S. Government Printing Office, Washington, DC.

Atkinson, D.H. and Gwynne, O. (1992) Design considerations for a Mars solar energy system. J. Brit. Interplanet. Soc. 45, 183-194.

Badescu, V. (1998) Different strategies for maximum solar radiation collection on Mars surface. Acta Astronautica 43, 409-421.

Blume, W.H. (1986) Computing the fictitious mean Sun for Mars and defining Martian time scales, JPL IOM 312/85.5-2397. Jet Propulsion Laboratory, Pasadena.

Bretagnon, P. and Francou, G. (1988) Planetary theories in rectangular and spherical variables: VSOP87 solutions, Astron. Astrophys. 202, 309-315.

Bretagnon, P. and Simon, J-L. (1986) Planetary Programs and Tables from -4000 to +2800 . Willmann-Bell, Inc., Richmond, VA.

Colburn, D.S., Pollack, J.B. and Haberle, R.M. (1989) Diurnal variations in optical depth at Mars. Icarus 79, 159-189.

Comité International de Poids et Mesures (1957) Proces Verbaux des Séances, deuxieme série 25, 77.

Davies, M.E., Abalakin, V.K., Bursa, M., Lieske, J.H., Morando, B., Morrison, D., Seidelmann, P.K., Sinclair, A.T., Yallop, B., Tjuflin, Y.S. (1996) Report of the IAU/AG/COSPAR working group on cartographic coordinates and rotational elements of the planets and satellites: 1994. Celest. Mech. Dynam. Astron. 63, 127-148.

Davies, M.E., Colvin, T., Kirk, R., Lee, E., and Sucharski, R. (1999) Status of the Rand-USGS control network of Mars. In The Fifth International Conference on Mars, Abstract \#6049. LPI Contribution No. 972, Lunar and Planetary Institute, Houston (CD-ROM).

Explanatory Supplement to the Astronomical Ephemeris and the American Ephemeris and Nautical Almanac. (1961) Her Majesty's Stationery Office, London.

Folkner, F.M., Yoder, C.F., Yuan, D.N., Standish, E.M. and Preston, R.A. (1997). Interior structure and seasonal mass redistribution of Mars from radio tracking of Mars Pathfinder. Science 278, 1749-1752.

François, L.M., Walker, J.C.G., and Kuhn, W.R. (1990) A numerical simulation of climate changes during the obliquity cycle on Mars. J.Geophys. Res. 95, $14,761-14,778$. 
Gangale, T. (1997) Mare Chronium: A short history of Martian timekeeping. In The Case for Mars IV: The International Exploration of Mars Considerations for Sending Humans, ed. T.R. Meyer. Univelt,Inc.; San Diego.

Glasstone, S. (1968) The Book of Mars. NASA SP-179. National Aeronautics and Space Administration. Washington, DC.

Golombek, M.P., Cook, R.A., Economou, T., Folkner, W.M., Haldemann, A.F.C., Kallemeyn, P.H., Knudsen, J.M., Manning, R.M., Moore, H.J., Parker, T.J., Rieder, R., Schofield, J.T., Smith, P.H., and Vaughan, R.M. (1997) Overview of the Mars Pathfinder mission and assessment of landing site predictions. Science 278, 174:-1748.

Green, R.M. (1985). Spherical Astronomy. Cambridge Univ. Press, Cambridge. Hartmann, W.K. (1997). Mars Underground. Tor Books, New York.

Harvey, D.A. (1982) The analemmas of the planets, Sky and Tel. 63, 237-239.

Hatcher, D.A. (1984) Simple formulae for Julian Day Numbers and calendar dates. Q.J. Roy. Astron. Soc. 25, 53-55.

Hilton, J.L. (1991) The motion of Mars' pole. I. Rigid body precession and nutation. Astron. J. 102, 1510-1527.

Hohenkerk, C.Y., Yallop, B.D., Smith, C.A., and Sinclair, A.T. Celestial reference systems. In Explanatory Supplement to the Astronomical Almanac, ed. P.K. Seidelmann. University Science Books, Mill Valley.

International Astronomical Union. (1998) Resolution B1: On the use of Julian Dates. IAU Information Bulletin 81, 29-30.

Kaplan, D. (1988) Environment of Mars, 1988. NASA TM 100470. National Aeronautics and Space Administration; Washington, DC.

Lee, W. (1995) Mars fictitious mean sun and equation of time. JPL IOM 312/95.5-4353. Jet Propulsion Laboratory, Pasadena.

Levine, J.S., Kraemer, D.R., and Kuhn, W.R. Solar radiation incident on Mars and the other planets: latitudinal, seasonal, and atmospheric effects. Icarus 31, 136-145.

Lowell (1905) Mon. Not. Roy. Astron. Soc. 66, 56.

Markowitz, W., Hall, R.G., Essen, L., and Perry, J.V.L. (1958) Frequency of cesium in terms of Ephemeris Time. Phys. Rev. Lett. 1, 105-107.

Meeus, J. (1964) The Martian Calendar. Spaceflight 6 (1), 30.

Meeus, J. (1991) Astronomical Algorithms, Willmann-Bell, Richmond.

Meeus, J. (1995) Astronomical Tables of the Sun, Moon and Planets, WillmannBell, Richmond.

Michael, W.H., Jr. (1979) Viking Lander tracking contributions to Mars mapping. Moon and Planets 20, 149-152.

Michaux, C.M. and Newburn, Jr., R.L. (1972). Mars Scientific Model. JPL Document 606-1 (NASA-CR-130711) Jet Propulsion Laboratory, Pasadena. 
Newcomb, S. (1895) Tables of the motion of the earth on its axis and around the Sun. Astronomical Papers of the American Ephemeris. 6, 1-169.

Roy, A.E. (1982). Orbital Motion. Adam Hilger, Ltd., Bristol.

Seidelmann, P.K. and T. Fukushima (1992) Why new time scales? Astron. Astrophys. 265 833-838.

Simon, J.L., Bretagnon, P., Chapront, J., Chapront-Touzé, M., Francou, G. and Lasker, J. (1994). Numerical expressions for precession formulae and mean elements for the Moon and planets. Astron. and Astrophys. 282, 663-683.

Smart, W.A. (1962)Text-Book on Spherical Astronomy, Cambridge Univ. Press. Snyder, C. (1977) The missions of the Viking Orbiters. J. Geophys. Res. 82, 3971-3983.

Soma, M. and Aoki, S. (1990) Transformation from FK4 system to FK5 system. Astron. and Astrophys. 240, 150-158.

Standish, E.M., Newhall, X.X., Williams, J.G., and Yeomans, D.K. (1992) Orbital Ephemerides of the Sun, Moon, and Planets. In Explanatory Supplement to the Astronomical Almanac, ed. P.K. Seidelmann. University Science Books, Mill Valley.

Sturms, F.M., Jr. (1970). Polynomial expressions for planetary equators and orbit elements with respect to the mean 1950.0 coordinate system. JPL Technical Memorandum 392-28. Jet Propulsion Laboratory, Pasadena.

Surán, J. (1997). A calendar for Mars. Planet. Space Sci. 45, 705-708.

Taff, L.G. (1985) Celestial Mechanics: A Computational Guide for the Practitioner, John Wiley, New York.

Tillman, J.E., Johnson, N.C., Guttorp, P., Percival, D.B. (1993). The Mars annual atmospheric pressure cycle: Years without great dust storms. J. Geophys. Res. 98, 10963-10971.

U.S. Naval Observatory. Multiyear Interactive Computer Almanac 1990-2005. (1998) Willmann-Bell, Inc., Richmond, VA.

Van Flandern, T.C. and Pulkkinen, K.F. (1979) Low-precision formulae for planetary positions. Astrophys. J. Supp. Series 41, 391-411.

Woolard, E.W. and Clemence, G.M. (1966) Spherical Astronomy. Academic Press, New York.

Yallop, B.D. and Hohenkerk, C.Y. (1992) Astronomical Phenomena. In Explanatory Supplement to the Astronomical Almanac, ed. P.K. Seidelmann. University Science Books, Mill Valley.

Yoder, C.F. and E.M. Standish (1997) Martian precession and rotation from Viking lander range data. J. Geophys. Res. 102, 4065-4080.

Zurek, R.W. and Martin, L.J. (1993). Interannual variability of planetencircling dust storms on Mars. J. Geophys. Res. 98, 3247-3259. 


\section{Appendix: Mars Season and Perihelion Dates}

The accompanying table lists the Modified Julian Date for each instance of the vernal equinox, northern summer solstice, autumnal equinox, and northern winter solstice, along with the perihelion passage for each of 135 orbits of Mars spanning the years 1874-2127. These were calculated from the truncated VSOP87 representation tabulated by Meeus (1991), also using the new Mars pole vector derived from Pathfinder radio tracking data by Folkner et al. (1997) for the evaluation of the seasonal passages. The Modified Julian Date, equivalent to the Julian Date minus 2400000.5, affords the advantage of a shorter 5-digit representation of the day number as compared with the 7-digit string of a Julian Day number and has been recommended by the International Astronomical Union (1998) for use where it is convenient to employ a day beginning at midnight (rather than Greenwich noon). Conversions to/from the MJD or JD and a given date in the terrestrial calendar may be determined from any of several established algorithms (e.g. Van Flandern and Pulkkinen, 1979; Hatcher, 1984), with 2000 Jan 1.0 = MJD 51544.0.

The tabulated seasonal dates may be compared with those given by Meeus (1995), as calculated with the older IAU Mars pole vector, and including an adjustment for the Earth-Mars light-travel time. Although the seasonal passages have been tabulated to the nearest thousandth day, the $0^{\circ} .001$ accuracy of the truncated VSOP87 representation (Meeus, 1991) used for their evaluation may admit to errors occasionally as large as $\pm 0.002 \mathrm{~d}$. Perihelion dates have been rounded to the nearest hundredth day, consistent with an estimated 5 times larger $\left(\sim 0^{\circ} .005\right)$ maximum error for their angular placement.

The tabulated Mars revolution number corresponds to a count of successive orbits (of 1.88083 Julian Years) post-1874.0. The adopted orbital epoch precedes the first observation of a Mars regional dust storm during the perihelic opposition of 1877 ( $c f$. Zurek and Martin, 1993) within the indicated "Rev 1", as well as the hand-drawn maps of the same era bearing the first antecedents for the modern cartographic nomenclature ( $c f$. Glasstone, 1968). By the same reckoning, J2000 occurs approximately 6 days prior to the start of Rev 67 . 
Table A1. Mars equinox, solstice, and perihelion dates.

\begin{tabular}{|c|c|c|c|c|c|c|}
\hline $\begin{array}{c}\text { Revs } \\
\text { post- } \\
1874.0\end{array}$ & $\begin{array}{c}\text { Julian Year } \\
\text { Interval }\end{array}$ & $\begin{array}{l}\text { Modified } \\
\text { Vernal } \\
\text { Equinox }\end{array}$ & $\begin{array}{l}\text { Julian Date } \\
\text { N.Summer } \\
\text { Solstice }\end{array}$ & $\begin{array}{c}\mathrm{MJD}=\mathrm{JD}- \\
\text { Autumnal } \\
\text { Equinox }\end{array}$ & $\begin{array}{c}2400000.5) \\
\text { N.Winter } \\
\text { Solstice }\end{array}$ & $\begin{array}{l}\text { Mars } \\
\text { Peri- } \\
\text { helion }\end{array}$ \\
\hline 0 & & 5 & 78 & 5 & 6197 & 6165.88 \\
\hline 1 & 1875 & 6355.680 & 6554.453 & $673^{\prime}$ & 6884.078 & 6852.73 \\
\hline 2 & $1877-1879$ & 7042.670 & 7241.422 & 7424.526 & 7571.060 & 7539.80 \\
\hline 3 & $1879-1881$ & 7729.641 & 7928.391 & 8111.472 & 8258.049 & 8226.86 \\
\hline 4 & 1881 & 8416.619 & 8615.380 & 8798.441 & 8944.979 & 8913.61 \\
\hline 5 & 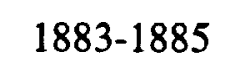 & 103 & 9302.345 & 948 & 9631.969 & 9600.75 \\
\hline 6 & 1885 & 9790 & 9989.291 & 1017 & 10318.974 & 10287.89 \\
\hline 7 & 1887 & 10477 & 10676.246 & 1085 & 11005.929 & 10974.73 \\
\hline 8 & 1889 & 11164 & 11363.218 & 1154 & 11692.879 & 11661.66 \\
\hline 9 & 1890 & 11851.446 & 12050.195 & 1223 & 12379.887 & 12348.85 \\
\hline 10 & & & 12737 & 1292 & 13066.853 & 13035.67 \\
\hline 11 & 1894 & 13225 & 13424.152 & 1360 & 13753.824 & 13722.69 \\
\hline 12 & 1896 & 13912 & 14111.104 & $142 S$ & 14440.832 & 14409.88 \\
\hline 13 & 0 & 14599 & 14798.058 & 149 & 15127.803 & 15096.80 \\
\hline 14 & $1900-$ & 15286.315 & 15485.026 & 15668 & 1581 & 15783.63 \\
\hline 15 & & 15973 & 16171.997 & 163 & & 16470.78 \\
\hline 16 & 5 & 16660 & 16858.973 & 170 & 1718 & 17157.66 \\
\hline 17 & & 173 & 17545.969 & 177 & 1787 & 17844.55 \\
\hline 18 & 99 & 18034.173 & 18232.933 & 1841 & 1856 & 18531.81 \\
\hline 19 & $1909-1911$ & 18721.147 & 18919.882 & 19103.091 & 19249.687 & 19218.85 \\
\hline 20 & & 194 & 19606 & 197 & 1993 & 19905.64 \\
\hline 21 & 5 & 200 & 20293.822 & 2047 & 20623.597 & 20592.70 \\
\hline 22 & 17 & 20782.081 & 20980.790 & 21163.978 & 21310.583 & 21279.73 \\
\hline 23 & $1917-1919$ & 21469.058 & 21667.775 & 2185 & 21997.519 & 21966.51 \\
\hline 24 & $1919-1921$ & 22156.017 & 22354.735 & 22537.965 & 22684.513 & 22653.68 \\
\hline 25 & & 22842.963 & 23041.686 & 232 & 23371.513 & 23340.80 \\
\hline 26 & & 23529.946 & 23728.655 & 2391 & 24058.485 & 24027.67 \\
\hline 27 & $1924-1926$ & 24216.936 & 24415.623 & 2459 & 24745.458 & 24714.65 \\
\hline 28 & 1926 & 24903. & 25102.590 & 2528 & 25432.453 & 25401.78 \\
\hline 29 & $1928-1930$ & 25590.883 & 25789.575 & 25972.792 & 26119.388 & 26088.54 \\
\hline
\end{tabular}




$\begin{array}{lllllll}30 & 1930-1932 & 26277.851 & 26476.649 & 26659.807 & 26806.362 & 26775.59 \\ 31 & 1932-1934 & 26964.791 & 27163.498 & 27346.783 & 27493.375 & 27462.78 \\ 32 & 1934-1936 & 27651.767 & 27850.452 & 28033 . .722 & 28180.343 & 28149.69 \\ 33 & 1936-1937 & 28338.761 & 28537.424 & 28720.696 & 28867.287 & 28836.53 \\ 34 & 1937-1939 & 29025.727 & 29224.405 & 29407.687 & 29554.288 & 29523.71 \\ & & & & & & \\ 35 & 1939-1941 & 29712.689 & 29911.387 & 30094.654 & 30241.272 & 30210.60 \\ 36 & 1941-1943 & 30399.689 & 30598.372 & 30781.659 & 30928.229 & 30897.52 \\ 37 & 1943-1945 & 31086.641 & 31285.328 & 31468.648 & 31615.237 & 31584.72 \\ 38 & 1945-1947 & 31773.600 & 31972.280 & 32155.592 & 32302.219 & 32271.72 \\ 39 & 1947-1949 & 32460.591 & 32659.244 & 32842.553 & 32989.166 & 32958.53 \\ & & & & & & \\ 40 & 1949-1951 & 33147.566 & 33346.212 & 33529.538 & 33676.143 & 33645.64 \\ 41 & 1951-1952 & 33834.524 & 34033.180 & 34216.482 & 34363.122 & 34332.62 \\ 42 & 1952-1954 & 34521.504 & 34720.174 & 34903.471 & 35050.060 & 35019.43 \\ 43 & 1954-1956 & 35208.456 & 35407.144 & 35590.500 & 35737.080 & 35706.65 \\ 44 & 1956-1958 & 35895.414 & 36094.088 & 36277.459 & 36424.091 & 36393.76 \\ & & & & & & \\ 45 & 1958-1960 & 36582.415 & 36781.048 & 36964.400 & 37111.039 & 37080.58 \\ 46 & 1960-1962 & 37269.398 & 37468.021 & 37651.382 & 37797.994 & 37767.55 \\ 47 & 1962-1964 & 37956.351 & 38154.988 & 38338.343 & 38484.989 & 38454.67 \\ 48 & 1964-1966 & 38643.323 & 38841.971 & 39025.306 & 39171.928 & 39141.44 \\ 49 & 1966-1968 & 39330.293 & 39528.942 & 39712.319 & 39858.909 & 39828.52 \\ & & & & & & \\ 50 & 1968-1969 & 40017.237 & 40215.896 & 40399.290 & 40545.917 & 40515.68 \\ 51 & 1969-1971 & 40704.215 & 40902.864 & 41086.248 & 41232.895 & 41202.59 \\ 52 & 1971-1973 & 41391.209 & 41589.836 & 41773.242 & 41919.863 & 41889.49 \\ 63 & 1992-1994 & 48947.868 & 49146.482 & 49329.956 & 49476.625 & 49446.65 \\ 53 & 1994-1996 & 49634.854 & 49833.442 & 50016.907 & 50163.575 & 50133.47\end{array}$




\begin{tabular}{|c|c|c|c|c|c|c|}
\hline 5 & $1996-1998$ & 50321.836 & 50520.413 & 50703.893 & 50850.540 & 50820.48 \\
\hline & $1998-2000$ & 51008.795 & 51207.382 & 51390.848 & 51537.531 & 51507.56 \\
\hline & $2000-2001$ & 1695.773 & 1894.376 & 52077.819 & 52224.466 & 52194.32 \\
\hline & 2001-2003 & 52382.737 & 52581.358 & 52764.856 & 52911.471 & 52881.46 \\
\hline & 003-2005 & 53069.686 & 53268.306 & 53451.834 & 53598.497 & 3568.65 \\
\hline & 2005-2007 & 53756.684 & 53955.264 & 54138.774 & 5428 & 54255.52 \\
\hline & $2007-2009$ & 54443.676 & 54642.236 & 54825.750 & 54972.406 & 54942.41 \\
\hline & 2009-2011 & 55130.635 & 55329.204 & 55512.721 & 55659.403 & 55629.58 \\
\hline & $2011-2013$ & 55817.601 & 56016.182 & 56199.670 & 56346.353 & 56316.38 \\
\hline & 2013-2015 & 56504.580 & 56703.159 & 56886.679 & 57033.314 & 57003.35 \\
\hline & 2015-2016 & 7191.524 & 57390.111 & 3.661 & 23 & 7690.55 \\
\hline & 2016-2018 & 57878.490 & 58077.073 & 58260.613 & 306 & 58377.53 \\
\hline & 2018-2020 & 58565.481 & 58764.042 & 58947.600 & 272 & 59064.38 \\
\hline & $2020-2022$ & 59252.467 & 59451.011 & 59634.581 & 597 & 59751.54 \\
\hline & $2022-2024$ & 59939.430 & 60137.983 & 60321.519 & 226 & 60438.45 \\
\hline & 2024-2026 & 60626.407 & 60824.969 & 13 & 67 & 61125.30 \\
\hline & $2026-2028$ & 61313.354 & 61511.924 & 5.515 & 76 & 61812.51 \\
\hline & $2028-2030$ & 62000.309 & 62198.873 & 2.461 & 169 & 62499.55 \\
\hline 3 & $2030-2031$ & 62687.304 & 62885.838 & 9.414 & 112 & 63186.34 \\
\hline & $2031-2033$ & 63374.285 & 63572.820 & 63756.409 & .083 & 63873.39 \\
\hline & $33-2035$ & 64061.239 & 64259.801 & & & 64560.49 \\
\hline & $2035-2037$ & 64748.227 & 64946.785 & 30.369 & 045 & 65247.28 \\
\hline & $2037-2039$ & 65435.203 & 65633.752 & 7.377 & 37 & 65934.43 \\
\hline & 2039-2041 & 66122.148 & 66320.702 & 04.335 & 40 & 66621.56 \\
\hline & $2041-2043$ & 66809.131 & 67007.659 & 67191.279 & 7.998 & 67308.41 \\
\hline & $43-2045$ & 67496.121 & $0 \%$ & & & 67995.33 \\
\hline & $2045-2047$ & 68183.082 & 68381.596 & 24 & 45 & 68682.47 \\
\hline 92 & $2047-2048$ & 68870.052 & 69068.581 & 52.178 & 886 & 69369.25 \\
\hline 93 & $2048-2050$ & 69557.024 & 69755.568 & 69939.209 & 70085.868 & 70056.30 \\
\hline 94 & $2050-2052$ & 70243.964 & 70442.515 & 70626.202 & 70772.900 & 70743.53 \\
\hline & $2052-2054$ & 70930.952 & 71129.467 & & & 71430.47 \\
\hline 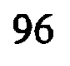 & $2054-2056$ & 71617.948 & 71816.435 & 77200.101 & 72146.812 & 72117.30 \\
\hline 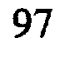 & $2056-2058$ & 72304.911 & 72503.405 & 72687.080 & 72833.799 & 72804.45 \\
\hline & $2058-2060$ & 72991.869 & 73190.377 & 73374.025 & 73520.764 & 73491.33 \\
\hline & $2060-2062$ & 351 & 77.362 & 74061.025 & 4207 & \\
\hline
\end{tabular}




\begin{tabular}{|c|c|c|c|c|c|c|}
\hline 100 & $2062-2063$ & 74365.799 & 74564.320 & 74748.023 & 74894.717 & 74865.41 \\
\hline 101 & $2063-2065$ & 75052.757 & 75251.281 & 75434.980 & 75581.712 & 75552.45 \\
\hline 102 & $2065-2067$ & 75739.747 & 75938.252 & 76121.962 & 76268.684 & 76239.30 \\
\hline 103 & $2067-2069$ & 76426.742 & 76625.222 & 76808.951 & 76955.667 & 76926.39 \\
\hline 104 & $2069-2071$ & 77113.709 & 77312.193 & 77495.895 & 77642.650 & 77613.41 \\
\hline 105 & $2071-2073$ & 77800.688 & 77999.183 & 78182.873 & 78329.583 & 78300.18 \\
\hline 106 & 2073-2075 & 78487.646 & 78686.146 & 78869.884 & 79016.582 & 78987.35 \\
\hline 107 & $2075-2077$ & 79174.594 & 79373.094 & 79556.840 & 79703.585 & 79674.45 \\
\hline 108 & 2077-2079 & 79861.583 & 80060.053 & 80243.785 & 80390.535 & 80361.28 \\
\hline 109 & $2079-2080$ & 80548.570 & 80747.030 & 80930.772 & 81077.491 & 81048.24 \\
\hline 110 & $2080-2082$ & 81235.524 & 81434.009 & 81617.756 & 81764.505 & 81735.41 \\
\hline 111 & $2082-2084$ & 81922.500 & 82120.987 & 82304.722 & 82451.461 & 82422.21 \\
\hline 112 & $2084-2086$ & 82609.486 & 82807.959 & 82991.729 & 83138.435 & 83109.27 \\
\hline 113 & $2086-2088$ & 83296.427 & 83494.908 & 83678.697 & 83825.440 & 83796.44 \\
\hline 114 & $2088-2090$ & 83983.399 & 84181.861 & 84365.637 & 84512.406 & 84483.35 \\
\hline 115 & $2090-2092$ & 84670.390 & 84868.829 & 85052.610 & 85199.352 & 85170.20 \\
\hline 116 & $2092-2094$ & 85357.354 & 85555.799 & 85739.588 & 85886.342 & 85857.35 \\
\hline 117 & $2094-2095$ & 86044.317 & 86242.777 & 86426.536 & 86573.299 & 86544.20 \\
\hline 118 & $2095-2097$ & 86731.295 & 86929.772 & 87113.556 & 87260.264 & 87231.15 \\
\hline 119 & 2097-2099 & 87418.239 & 87616.727 & 87800.570 & 87947.300 & 87918.40 \\
\hline 120 & $2099-2101$ & 88105.221 & 88303.678 & 88487.510 & 88634.290 & 88605.41 \\
\hline 121 & $2101-2103$ & 88792.223 & 88990.646 & 89174.466 & 89321.231 & 89292.21 \\
\hline 122 & $2103-2105$ & 89479.197 & 89677.621 & 89861.451 & 90008.206 & 89979.29 \\
\hline 123 & $2105-2107$ & 90166.153 & 90364.590 & 90548.400 & 90695.188 & 90666.28 \\
\hline 124 & $2107-2109$ & 90853.135 & 91051.578 & 91235.385 & 91382.126 & 91353.08 \\
\hline 125 & $2109-2110$ & 91540.092 & 91738.539 & 91922.393 & 92069.125 & 92040.26 \\
\hline 126 & $2110-2112$ & 92227.041 & 92425.496 & 92609.353 & 92756.128 & 92727.36 \\
\hline 127 & $2112-2114$ & 92914.027 & 93112.465 & 93296.324 & 93443.101 & 93414.23 \\
\hline 128 & $2114-2116$ & 93601.024 & 93799.432 & 93983.311 & 94130.072 & 94101.23 \\
\hline 120 & $2116-2118$ & 94287.992 & 94486.400 & 94670.263 & 94817.062 & 94788.34 \\
\hline 130 & $2118-2120$ & 94974.963 & 95173.385 & 95357.222 & 95503.994 & 95475.09 \\
\hline 31 & $2120-2122$ & 95661.928 & 95860.354 & 96044.237 & 96190.974 & 96162.18 \\
\hline 32 & 2122-2124 & 96348.866 & 96547.301 & 96731.206 & 96877.984 & 96849.35 \\
\hline 33 & $2124-2126$ & 97035.845 & 97234.255 & 97418.146 & 97564.947 & 97536.24 \\
\hline$?$ & $2126-2127$ & 97722.837 & 97921.228 & 98105.125 & 98251.894 & 98223.11 \\
\hline
\end{tabular}



$-\dot{*}=$ 